\title{
Electoral Platforms, Implemented Policies, and Abstention
}

\author{
Humberto Llavador* \\ Department of Economics \\ Universitat Pompeu Fabra \\ e-mail: humberto.llavador@upf.edu
}

October 27, 2004

\begin{abstract}
Recent studies of American politics evidence that political polarization of both the electorate and the political elite have moved "almost in tandem for the past half century" (McCarty et al., 2003, p.2), and that party polarization has steadily increased since the 1970s. On the other hand, the empirical literature on party platforms and implemented policies has consistently found an imperfect but nonnegligible correlation between electoral platforms and governmental policies: while platforms tend to be polarized, policies are moderate or centrist. However, existing theoretical models of political competition are not manifestly compatible with these observations.

In this paper, we distinguish between electoral platforms and implemented policies by incorporating a non-trivial policy-setting process. It follows that voters may care not only about the implemented policy but also about the platform they support with their vote. We find that while parties tend to polarize their positions, the risk of alienating their constituency prevents them from radicalizing. The analysis evidences that the distribution of the electorate, and not only the (expected) location of a pivotal voter, matters in determining policies. Our results are consistent with the observation of polarized platforms and moderate policies, and the alienation and indifference components of abstention.

Keywords: Platforms, Voting, Polarization, Abstention, Alienation.

JEL Classification: D72
\end{abstract}

*I am very grateful to Joaquim Silvestre, John Roemer, Giacomo Bonanno, Louis Makowski, Martine Quinzii, Carmen Beviá, Robert Oxoby, Ignacio Ortuño-Ortín, Antonio Cabrales, and two anonymous referees for their very helpful comments and suggestions. I thank seminar participants at the Econometric Society, ASSET, and ECPR meetings. Support from the Spanish Ministry of Education through grant SEC2000-1186, the Fundación Ramón Areces, and the Barcelona Economics Program of CREA are gratefully acknowledged. 


\section{Introduction}

In a recent study of American politics, McCarty et al. (2003) evidence that political polarization has steadily increased since the 1970s. There is similar evidence that the mass public is also increasingly polarized in terms of liberal-conservative views (Green et al., 2002). ${ }^{1}$ However, it is not manifest that the existing theoretical models of political competition are compatible with this divergency within the political elite and the electorate. Even those models whose equilibria present differentiated electoral platforms find that parties are subject to a centripetal force only palliated by bounded rationality (Kollman et al., 1992), valence issues (Ansolabehere and Snyder, 2000; Aragonés and Palfrey, 2002), incumbency advantages -and "valence" issues- (Bernhardt and Ingberman, 1985; Londregan and Romer, 1993), uncertainty and ideology (Wittman, 1977; Calvert, 1985), or internal decision-making (Snyder, 1994).

We believe that the critical element missing from the dominant literature is the distinction between electoral platforms and implemented policies and the fact that the outcome of an election affects the actions taken by the winners. That is, electoral victors do not implement the platform on which they were elected but rather temper their implement policies by their margin of victory. Supporting our claim, the empirical literature that examines the relationship between party platforms and implemented policies has consistently reported two observations. First, while platforms tend to be polarized, policies are more moderate or centrist. ${ }^{2}$ Secondly, although electoral platforms and governmental outcomes systematically differ from each other, they are not stochastically independent. ${ }^{3}$

In this paper, we develop a model of political competition which is consistent with these findings. We depart from the dominant literature by letting the implemented policy differ systematically from the electoral platform of the winning party. In particular, following the spirit of the empirical observations, the implemented policy will depend on the voting outcome and on the platforms announced by each party. Thus, there exists a process of policy setting in which the higher the fraction of votes obtained by a party, the closer the implemented policy is to its proposal. We

\footnotetext{
${ }^{1}$ For instance, using data from Green et al. (2002), McCarty et al. (2003) report that "the difference between the 'percentage of Republicans who call themselves conservatives' and the 'percentage of Democrats who call themselves conservatives' has doubled between 1972 and 1996, moving from $25 \%$ to $50 \%$." (p.3)

${ }^{2}$ Poole and Rosenthal (1984b) use thermometer scores to measure the perceived location of candidates and policies; Fiorina (1974), Poole and Rosenthal (1984a), Poole and Daniels (1985) use interest groups ratings; Poole and Rosenthal (1991, 1997), Snyder (1996), and Alesina and Rosenthal (1995) discover the same pattern using roll call votes in congress.

${ }^{3}$ See Budge and Hofferbert $(1990,1992)$, Hofferbert and Klingemann (1990), Klingermann et al. (1994), and King et al. (1993).
} 
do not require parties to be very sensitive to the electoral result, nor we impose any symmetric conditions in their post-electoral behavior. Thus, our representation will allow for "obstinate" candidates (who will implement a policy arbitrarily closed to their proposal even with a narrow margin of victory), as well as for very "reconciling" parties. Further, both types may co-exist in the same election. ${ }^{4}$

Modifying the policy-setting process has two important, and often overlooked, implications on voting behavior. First, unlike the winner-takes-all case, if platforms and implemented policies differ, voting for the preferred candidate is not a dominant strategy for all voters. Consider, for instance, a voter with moderate views who finds the proposal of the left party $(L)$ more appealing than that of the right party $(R)$. Suppose also that she expects, due to an overwhelming support for the left party, that the implemented policy will be too far left for her. Then, she may decide to vote for the right party in order to moderate the policy implemented by the left. That is, we may find 'strategic voting' in the sense that a voter who prefers the alternative offered by party $L$ to the one announced by $R$ may, nevertheless, vote for $R$ to moderate $L$ 's policy.

Second, since platforms and policies do not necessarily coincide, we cannot ignore that voters may consider both (the electoral platform and the effect on the implemented policy) when deciding whether to vote and for whom. For example, a conservative voter may find voting for an extreme-right party 'too costly', regardless of the impact that a larger support for that party would have on the implemented policy. Her reluctance would come not from the influence on final policies, but from the "ideology" represented by such a party. If the extreme-right party were the only alternative to obtain a more conservative policy, we may expect this voter to feel alienated and abstain. ${ }^{5}$

Therefore, it is necessary to distinguish two components on voting behavior. On the one hand, insofar as voters care about the policy outcome, they vote for the party that affects the implemented policy in the direction of their interests. This component represents the policy-oriented side of voters. On the other hand, voters face a "cost" of supporting a platform that differs from their most preferred policy. This is the platform-oriented side of voters. Because platforms and policies do not necessarily coincide, voters who would have supported a party from a purely policyoriented point of view may abstain because they strongly disagree with the party's platform. The final voting decision will depend then on both the policy-oriented and the platform-oriented sides of voters.

Our analysis incorporates the previous features and analyzes a two-party, unidimensional political competition game with a dynamic structure. In period one, parties announce their electoral platforms. In period two, citizens observe the plat-

\footnotetext{
${ }^{4}$ See Figure 2 for examples.

${ }^{5}$ See Rabinowitz and Macdonald (1989) for a similar voting behavior in a different context.
} 
forms and decide whether to vote for one of the two candidates or to abstain. Their decision is based on their preferences over policies and platforms and their expectations about the outcome of the election. Finally, after the election, a policy-setting process takes place in which the implemented policy depends on both the parties' platforms and their electoral support. We show that, in the absence of voting costs, parties polarize their positions, while it is the existence of voting costs that causes parties to moderate their platforms to avoid alienating the core of their constituency.

The paper is organized as follows. Section 2 describes the model and introduces the equilibrium concept. Readers interested in an overview of the main results can look first at the example in Section 3. The following two sections derive the formal results. Section 4 studies the case of costless voting. In equilibrium, everybody votes and parties radicalize their positions, yet the implemented policy is moderate and consistently differs from the median voter ideal policy. Section 5 incorporates a positive cost of voting. Parties announce differentiated yet non-extreme policies. Voters concentrate around the announced platforms, and substantial turnout generically obtains. Abstention occurs among voters with extreme views (those who feel alienated), as well as among voters with moderate views, who are sufficiently indifferent regarding marginal changes in the policy outcome. Section 6 concludes.

\section{The Model}

We model the electoral process as a political game between parties and voters with a dynamic structure. Two parties, $L$ and $R$, announce their campaign platforms by choosing a location in the policy space. Citizens observe the platforms and vote for one of the two candidates or abstain. After the election, a policy is implemented as a function of the electoral platforms and the voting outcome.

The following sections describe the three steps in the political game: platform announcement, voting, and policy formation. The timing of events is presented in Figure 1. We solve the game by backward induction.

The electoral arena is assumed to be represented by a unidimensional policy space $T=[\underline{t}, \bar{t}] \subset \mathbb{R}$. We will use $T^{2}=T \times T$. There are two parties, labelled $L$ and $R$. We differentiate between parties' platforms (the policies on which parties campaign) and the government's policy (the policy implemented by the the electoral victor). We will denote parties' platforms by $t_{L}$ and $t_{R}$.

\subsection{Policy Formation}

A key feature of the present model is that the implemented policy may differ from the electoral platform of the winner. Policy is assumed to be the result of a postelectoral process where both parties play a role relative to their electoral support. 


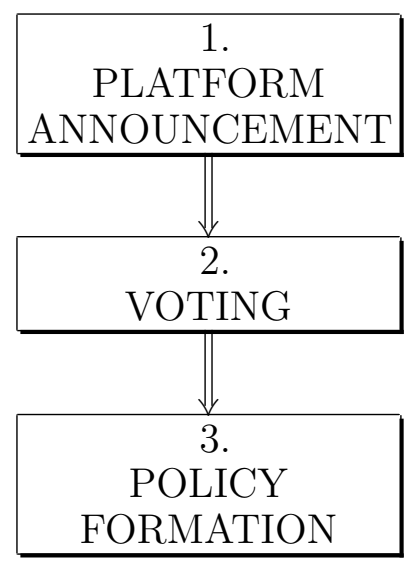

\section{PARTIES ANNOUNCE THE PLATFORMS} THEY WILL CAMPAIGN FOR.

\section{VOTERS VOTE FOR ONE} OF THE PARTIES OR ABSTAIN.

\section{A POLICY IS IMPLEMENTED AS A FUNCTION OF THE PLATFORMS AND THE ELECTORAL OUTCOME.}

Figure 1: Timing of events.

The literature on policy compromise have offered several explanations for the implemented policy to depend on the margin of victory. For example, Ortuño-Ortín (1997) argues that "a democratic society is integrated by different institutions and groups. Some of them will favor the policies announced by the winning party, some will favor the opposition's platforms, but all may have some influence on the actions taken by the government, and their influence will be a non-decreasing function of the support received by the corresponding party." Alternatively, Dixit et al. (2000) find tacit collusion between forward-looking parties in a plurality political environment. If we assume that the ruling party knows that its term will reach an end and expects that the other party will be in power sometime in the future, this party may then go to some extent towards the interests of the opposition when setting the policy, expecting the same treatment when it itself becomes the opposition party. A third argument considers the possibility that the opposition may decide to confront a policy and mobilize its constituency if it feels entitled to a better outcome. If confrontation (social unrest) is costly for the government, this may decide to deviate from its proposal and implement a more moderate policy (Ellman and Wantchekon, $2000)^{6}$

For the purpose of this paper, it will be convenient to abstract from the different stories and model policy setting in a reduce form by directly specifying a map from platforms and voting outcomes to final policies. In particular, we let the implemented policy be a function of the announced platforms $\left(t_{L}, t_{R}\right)$, and the voting outcome $(\nu, 1-\nu)$, where $\nu$ is the share of the vote that party $\mathrm{L}$ received. Let the

\footnotetext{
${ }^{6}$ Other justifications can be found in Austen-Smith and Banks (1998); Alesina and Rosenthal (1995, 1996, 2000); Gerber and Ortuño-Ortín (1998); Grossman and Helpman (1996); De Sinopoli and Iannantuoni (2003).
} 
weight function $g:[0,1] \rightarrow[0,1]$ such that $g(\nu)$ represents the weight of party $L$ 's proposal in the implemented policy. Then we define the implemented policy function. ${ }^{7}$

Definition 2.1 Let $t_{L}$ and $t_{R}$ be the platforms announced by party $L$ and party $R$, respectively. Let $\nu$ be the share of the vote cast for $L$. Then we define the implemented policy function $\hat{t}: T^{2} \times[0,1] \rightarrow T$ as

$$
\hat{t}\left(t_{L}, t_{R}, \nu\right)=g(\nu) t_{L}+(1-g(\nu)) t_{R}
$$

The following assumption describes the only conditions imposed on $g$.

$$
\begin{array}{ll}
\text { Assumption } 1 \text { (A1) a) } & g \in C^{1} \\
& \text { b) } \\
& g(0)=0, g(1)=1 ; \\
\text { c) } & g^{\prime}(\nu)>0, \quad \forall \nu \in(0,1) .
\end{array}
$$

Part $(b)$ implies that if one of the parties obtains all the votes, it implements its announced platform. By $(c)$, the larger the share of the vote a party obtains, the stronger its influence in the final policy. Finally, part $(a)$ introduces continuity: small perturbations in the voting outcome cannot produce large changes in the implemented policy.

Assumption 1 is common in this literature and allows for an implemented policy function $\hat{t}$ general enough to represent a wide variety of scenarios. Continuity is perhaps the most restrictive assumption, since it rules out games in which "the winner takes all". ${ }^{8}$ However, as shown in Figure 2(a), it is easy to find an almostwinner-takes-all type of function that satisfies A1, for which the winning party is able to implement a policy arbitrarily close to its electoral platform if it obtains a plurality larger than $\epsilon$. Our setting also allows for "obstinate" candidates (who will implement a policy arbitrarily close to their proposal even with a narrow margin of victory) as well as for very "reconciling" parties running in the same election (see Figure 2(b) for an example of such an asymmetric implemented function). ${ }^{9}$

\footnotetext{
${ }^{7}$ The implemented policy function is equivalent to the legislative outcome function in AustenSmith (1989), the implemented policy function in Ortuño-Ortín (1997), the outcome function in Gerber and Ortuño-Ortín (1998), or the policy outcome function in De Sinopoli and Iannantuoni (2003).

${ }^{8}$ Continuity could be relaxed by introducing a discontinuity at voting outcome $\nu=\frac{1}{2}$ without affecting the predictions of the model, except perhaps for equilibrium existence. Observe, however, that monotonicity, $\mathrm{A} 1(\mathrm{c})$, is critical: a larger share of the vote needs to translate into a more favorable implemented policy, excluding hence pure "winner-takes-all" systems.

${ }^{9}$ For the shake of presentation we have depicted all implemented policy functions going through the mid-point between $t_{L}$ and $t_{R}$, although this is not required by assumption 1 .
} 


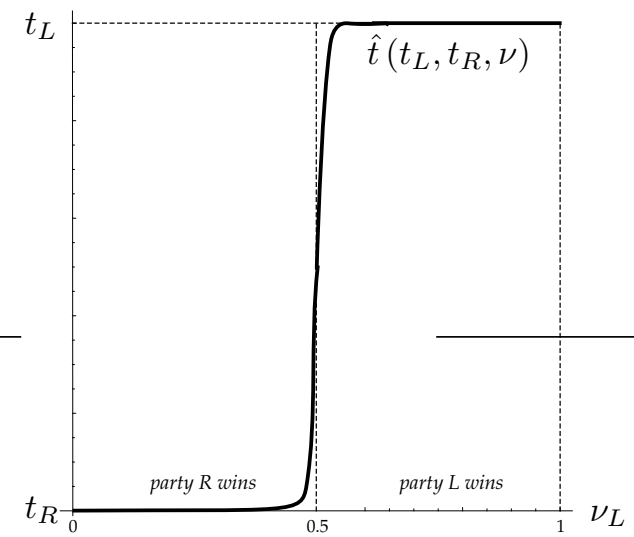

(a) The "almost" winner-takes-all function: the party with more than $(50+\epsilon) \%$ of the votes implements a policy arbitrarily close to its electoral platform.

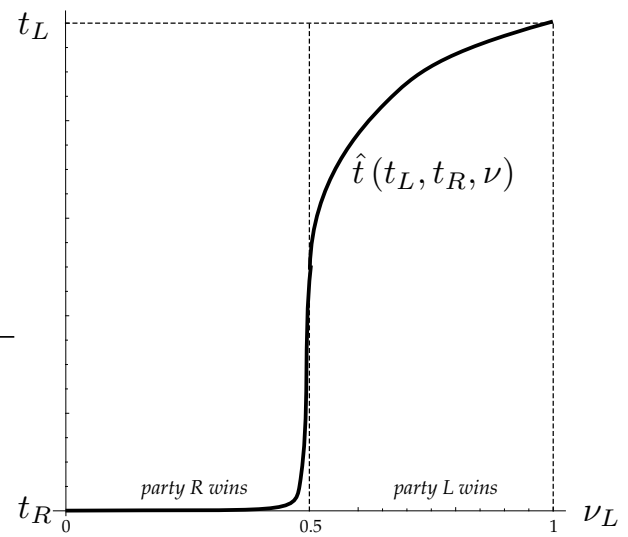

(b) A non-symmetric function: controlling for the share of the vote received, party $L$ is more sensitive to the electoral outcome than party $R$.

Figure 2: Examples of implemented policy functions. Given, $t_{L}$ and $t_{R}$, the function $\hat{t}$ assigns to the share of votes received by party $L$ the policy finally adopted. Assumption 1 allows for (a) almost discontinuous functions and (b) non-symmetric functions.

\subsection{Voting}

There is a continuum of citizens. Each citizen has preferences over policies represented by the concave utility function $u: T \rightarrow \mathbb{R}$, where $u(t ; \tau)$ represents the utility that a citizen of type $\tau$ obtains when $t$ is implemented. Citizens differ then with respect to their ideal policy. Let $F: \mathbb{R} \rightarrow[0,1]$ describe the distribution of citizens. We assume $F$ to be continuous and with a density function $f$.

Citizens observe the platforms announced by the parties, $t_{L}$ and $t_{R}$, and anticipate the policy-making process (i.e. they know the implemented policy function $\hat{t}$ and have expectations regarding the support that each party will receive.) With this information, each citizen decides between voting for $L$, voting for $R$, or abstaining. Let $S=\{L, R, A\}$ be the set of actions for a particular citizen, with typical element $s: s=L$ and $s=R$ denote that the citizen votes for party $L$ and party $R$, respectively; $s=A$ denotes abstention.

Insofar a voter cares about the policy outcome, she will choose to vote for a party based on the effect that greater support for that party has on the implemented policy. Because the implemented policy is a function of the electoral platforms and the allocation of votes, it is convenient to represent preferences as a function on platforms and electoral outcomes:

$$
\hat{u}\left(t_{L}, t_{R}, \nu ; \tau\right)=u\left(\hat{t}\left(t_{L}, t_{R}, \nu\right) ; \tau\right)
$$


Thus, citizen $\tau$ 's payoff from voting for $L$ will be the utility change implied by a larger support for party $L$. More precisely, for each pair of policies $\left(t_{L}, t_{R}\right)$ and an expected voting outcome $\nu, \tau$ 's benefit from voting for $L$ will be the partial derivative of $\tau$ 's utility over policies with respect to a change in the fraction of the vote received by party $L$. Observe that, because the fraction of voters for $R$ is $1-\nu$, an increase in the support for $R$ is equivalent to a decrease in the support for $L$. Finally, if a citizen abstains, her utility is equal to zero. Consequently, we define the benefit of voting, $w: S \times T^{2} \times[0,1] \times T \rightarrow \mathbb{R}$, as ${ }^{10}$

$$
w\left(s ; t_{L}, t_{R}, \nu, \tau\right)= \begin{cases}\frac{\partial \hat{u}\left(t_{L}, t_{R}, \nu ; \tau\right)}{\partial \nu} & \text { if } s=L \\ -\frac{\partial \hat{u}\left(t_{L}, t_{R}, \nu ; \tau\right)}{\partial \nu} & \text { if } s=R \\ 0 & \text { if } s=A\end{cases}
$$

As argued in the introduction, individuals may also care about the platform of the party they support with their vote. We will take that, independent from the effect on the implemented policy, a voter faces a 'cost' from voting for a platform that differs from her ideal policy. Let $\eta\left(t_{L} ; \tau\right)$ represent the cost that voter $\tau$ faces from voting for party $L$ when this party defends platform $t_{L}$. Similarly, let $\eta\left(t_{R} ; \tau\right)$ be her cost from voting for party $R$. Finally, normalize the cost of abstention to zero. Hence we can define the cost of voting as ${ }^{11}$

$$
\hat{\eta}\left(s ; t_{L}, t_{R}, \tau\right)= \begin{cases}\eta\left(t_{L} ; \tau\right) & \text { if } s=L \\ \eta\left(t_{R} ; \tau\right) & \text { if } s=R \\ 0 & \text { if } s=A\end{cases}
$$

It follows that a citizen will abstain whenever the cost of voting for her favorite party is higher than the corresponding benefit she obtains. On the other hand, if she decides to votes, she will support the party that yields the highest voting utility defined as:

$$
v\left(s ; t_{L}, t_{R}, \nu, \tau\right)=w\left(s ; t_{L}, t_{R}, \nu, \tau\right)-\hat{\eta}\left(s ; t_{L}, t_{R}, \tau\right) .
$$

Thus, given $t_{L}, t_{R}$ and $\nu$, we can partition the electorate into those citizens who

\footnotetext{
${ }^{10}$ This utility of voting is similar in spirit to Alesina and Rosenthal's (1995) conditional sincerity voting: voters vote with conditional sincerity if they vote "for the party whose legislative vote they would like to see increased" (p.50). Alternatively, the benefit of voting can also be justified as a limiting argument. Appendix A considers a society with a finite number of citizens $n$, and derives the benefit of voting as $n$ goes to infinity, obtaining expression 2.3 .

${ }^{11}$ Observe that this presentation would allow for a constant cost of votign by setting $\eta(t ; \tau)=k$ for all $t, \tau \in T$. Further, letting $k<0$ could represent a benefit of voting or a cost of abstention, in line with the "civil duty" arguments.
} 
vote for $L$, those who vote for $R$, and those who abstain according to

$$
\begin{aligned}
& L\left(t_{L}, t_{R}, \nu\right)=\left\{\tau \in T: v\left(L ; t_{L}, t_{R}, \nu, \tau\right) \geq \max \left\{0, v\left(R ; t_{L}, t_{R}, \nu, \tau\right)\right\}\right\} \\
& R\left(t_{L}, t_{R}, \nu\right)=\left\{\tau \in T: v\left(R ; t_{L}, t_{R}, \nu, \tau\right)>\max \left\{0, v\left(L ; t_{L}, t_{R}, \nu, \tau\right)\right\}\right\} \\
& A\left(t_{L}, t_{R}, \nu\right)=\left\{\tau \in T: \max \left\{v\left(L ; t_{L}, t_{R}, \nu, \tau\right), v\left(R ; t_{L}, t_{R}, \nu, \tau\right)\right\}<0\right\} .
\end{aligned}
$$

Let $V_{J}\left(t_{L}, t_{R}, \nu\right)=\int_{J\left(t_{L}, t_{R}, \nu\right)} d F$ be the fraction of the citizenry who votes for party $J$. Hence, $V_{L}+V_{R}$ represents the turnout rate and a fraction $1-V_{L}-V_{R}$ of the citizenry abstains.

We have described how citizens vote (or abstain) as a function of the electoral platforms and the expected support for each party. However, our goal is to find a correspondence that assigns to each pair of platforms the set of all consistent or rational-expectations voting outcomes, according to the following definition.

Definition 2.2 We say that $\nu$ is a consistent vote for the pair of electoral platforms $_{L}, t_{R}$ in $T$ if

$$
\frac{V_{L}\left(t_{L}, t_{R}, \nu\right)}{V_{L}\left(t_{L}, t_{R}, \nu\right)+V_{R}\left(t_{L}, t_{R}, \nu\right)}=\nu
$$

that is, if the allocation of votes implied when $\nu$ is expected gives rise to a fraction of votes for $L$ equal to $\nu$.

Thus, we can define the voting outcome correspondence $\chi: T^{2} \rightrightarrows 2^{[0,1]}$ as,

$$
\chi\left(t_{L}, t_{R}\right)=\left\{\nu \in[0,1]: \nu \text { is a consistent vote for }\left(t_{L}, t_{R}\right)\right\} .
$$

Perhaps abusing language, and without further specifications, this definition admits the possibility that $\chi\left(t_{L}, t_{R}\right)=\emptyset$ for some $t_{L}$ and $t_{R}$. However, in what follows, we will restrict the analysis to environments where $\chi\left(t_{L}, t_{R}\right) \neq \emptyset, \forall\left(t_{L}, t_{R}\right)$.

\subsection{Political Equilibrium}

Parties are the actual players of the political game, each having single-peaked preferences over policies represented by the utility function $\pi_{J}: T \rightarrow \mathbb{R}, J=L, R .^{12}$ Let $\tau_{J}=\arg \max \left\{\pi_{J}(t): t \in T\right\}$ be the ideal policy of party $J .{ }^{13}$ Without loss of generality, assume that $\tau_{L}<\tau_{R}$.

\footnotetext{
${ }^{12}$ Formally, given that $T$ is compact, single-peakedness of preferences is equivalent to the strict quasi-concavity of the utility function (Roemer, 2001, p.18).

${ }^{13}$ Observe that the preferences of a party do not need to coincide with those of a particular voter, even if they share the same ideal point. Also, it is worth emphasizing that we do not need to assume that voters know parties' preferences or ideal policies.
} 
It is convenient to write the utility of a party as a function of the platforms and the vote allocation using the definition of the implemented policy function. Define $\hat{\pi}_{J}: T^{2} \times[0,1] \rightarrow \mathbb{R}$ as

$$
\hat{\pi}_{J}\left(t_{L}, t_{R}, \nu\right)=\pi_{J}\left(\hat{t}\left(t_{L}, t_{R}, \nu\right)\right)
$$

For illustrative purposes, assume there exists a unique consistent voting outcome for each pair of policies, (that is, $\chi$ is a function). We can then write $\tilde{\pi}_{J}\left(t_{L}, t_{R}\right)=\hat{\pi}_{J}\left(t_{L}, t_{R}, \chi\left(t_{L}, t_{R}\right)\right)$ and a political equilibrium is simply a Nash equilibrium of the two-party game where parties choose platforms in $T$ to maximize payoffs $\tilde{\pi}_{J}$. In general, however, $\chi$ may not be single-valued and non-empty for all pairs of policies. Therefore we introduce the following more general definition of a political equilibrium:

Definition 2.3 We say that $\left(t_{L}, t_{R}, \bar{\nu}\right)$ is a political equilibrium if

(i) $\bar{\nu} \in \chi\left(t_{L}, t_{R}\right)$,

(ii) $\hat{\pi}_{L}\left(t_{L}, t_{R}, \bar{\nu}\right) \geq \hat{\pi}_{L}\left(t, t_{R}, \nu\right) \forall t \in T$ and $\forall \nu \in \chi\left(t, t_{R}\right)$, and

(iii) $\hat{\pi}_{R}\left(t_{L}, t_{R}, \bar{\nu}\right) \geq \hat{\pi}_{R}\left(t_{L}, t, \nu\right) \forall t \in T$ and $\forall \nu \in \chi\left(t_{L}, t\right)$.

That is, in equilibrium, there is no possible voting outcome for which a party can increase its payoff by announcing a different platform. Therefore, the definition represents situations where no party has an incentive to deviate from its equilibrium strategy.

\section{Example}

Before presenting the formal analysis, we anticipate the main results with an illustrative example.

Consider a political process where two ideological parties, $L$ and $R$, compete over a single issue. Let $T=[0,1]$ represent the policy space. The weight-of-votes function $g$ takes the following form (see Figure 3):

$$
g(\nu)=\frac{1-\cos (\pi \nu)}{2}
$$

Because $g$ is concave for $\nu>\frac{1}{2}$ and convex for $\nu<\frac{1}{2}$, winning the election makes a difference: the votes received by the government weight more in the policy-setting process than those received by the opposition. 


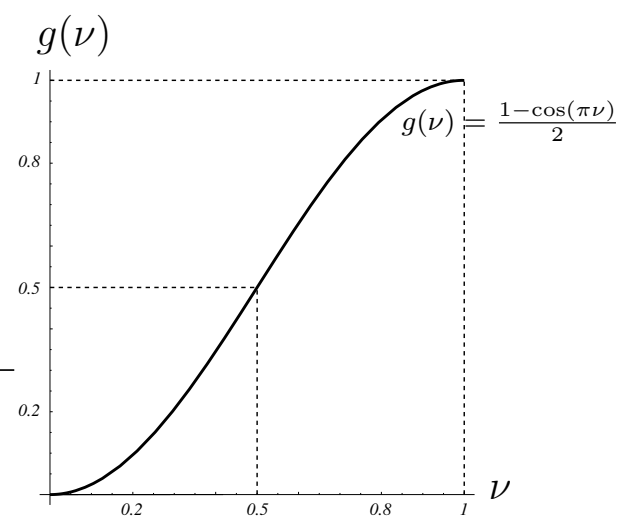

Figure 3: Weight of Votes Function.

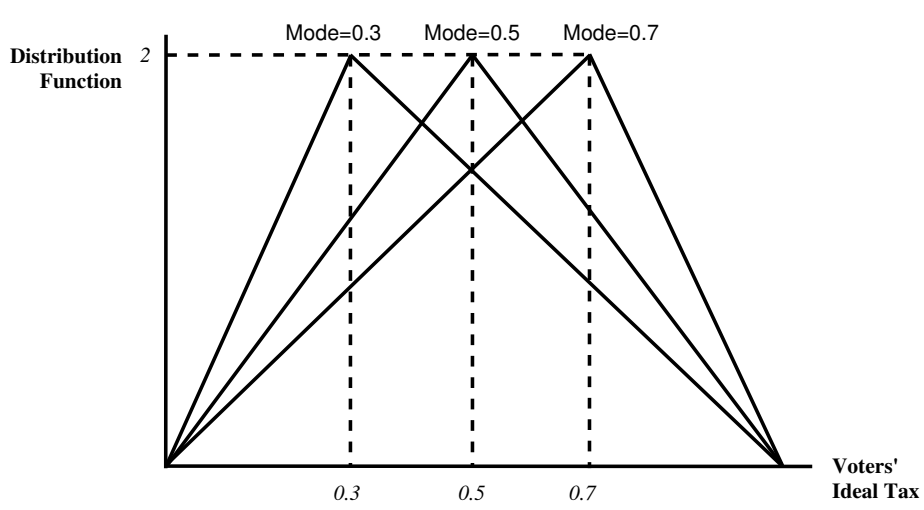

Figure 4: Family of Triangular Density Functions.

Let the electorate be distributed according to a triangular distribution with mode $m$ (see Figure 4 for a few members of this family of density functions). Voters' preferences over policies are represented by the following Euclidean utility function:

$$
u(t ; \tau)=-\frac{1}{2}(t-\tau)^{2}
$$

The benefit of voting is given by

$$
\begin{aligned}
& w\left(L ; t_{L}, t_{R}, \nu, \tau\right)=\left(\hat{t}\left(t_{L}, t_{R}, \nu\right)-\tau\right) g^{\prime}(\nu)\left(t_{R}-t_{L}\right) \\
& w\left(R ; t_{L}, t_{R}, \nu, \tau\right)=\left(\tau-\hat{t}\left(t_{L}, t_{R}, \nu\right)\right) g^{\prime}(\nu)\left(t_{R}-t_{L}\right)
\end{aligned}
$$

Voting costs represent the "dis-utility" of supporting a platform far from one's ideal policy. In particular,

$$
\eta(t ; \tau)=2|t-\tau| .
$$


Thus, the utility of voting of a citizen $\tau$ is

$$
\begin{aligned}
& v\left(L ; t_{L}, t_{R}, \nu, \tau\right)=\left(\hat{t}\left(t_{L}, t_{R}, \nu\right)-\tau\right) g^{\prime}(\nu)\left(t_{R}-t_{L}\right)-2\left|t_{L}-\tau\right|, \\
& v\left(R ; t_{L}, t_{R}, \nu, \tau\right)=\left(\tau-\hat{t}\left(t_{L}, t_{R}, \nu\right)\right) g^{\prime}(\nu)\left(t_{R}-t_{L}\right)-2\left|t_{R}-\tau\right|, \\
& v\left(A ; t_{L}, t_{R}, \nu, \tau\right)=0 .
\end{aligned}
$$

Parties have Euclidean preferences represented by

$$
\pi_{J}(t)=-\frac{1}{2}\left(t-\tau_{J}\right)^{2}
$$

We take the ideal policy of the left party to be $\tau_{L}=0.2$ and that of the right party to be $\tau_{R}=0.75$.

It is easy to show that, given $\left(t_{L}, t_{R}, \nu\right)$, voting occurs on two intervals around the electoral platforms: $\left[\lambda_{1}, \lambda_{2}\right] \ni t_{L}$ and $\left[\rho_{1}, \rho_{2}\right] \ni t_{R} \cdot{ }^{14}$ Hence,

$$
\begin{aligned}
V_{L}\left(t_{L}, t_{R}, \nu\right) & =F\left(\lambda_{2}\right)-F\left(\lambda_{1}\right), \\
V_{R}\left(t_{L}, t_{R}, \nu\right) & =F\left(\rho_{2}\right)-F\left(\rho_{1}\right) .
\end{aligned}
$$

We study two cases: (a) a symmetrically distributed electorate (i.e. $m=\frac{1}{2}$ ), and $(b)$ a right-skewed distribution $(m=0.7)$. Figure 5 , where we have represented the political equilibrium for each case, summarizes the main findings of the paper.

(i) Parties announce differentiated, non-extreme platforms. In the symmetric case, they locate symmetrically with respect to the mean. In the nonsymmetric case, they respond to the right-skewness of the distribution of the electorate and shift their platforms to the right. Platforms may be more moderate $\left(\tau_{L}=0.2<t_{L}^{*}=0.28\right)$ or more radical $\left(\tau_{R}=0.75<t_{R}^{*}=0.8\right)$ than parties' ideal policies.

(ii) The equilibrium policy does not coincide generically with the ideal policy of the median voter, except when the electorate is symmetrically distributed. Observe that the implemented policy also responds in the right direction to the skewness of the population.

\footnotetext{
${ }^{14}$ Of course, $\lambda_{1}, \lambda_{2}, \rho_{1}$, and $\rho_{2}$ are functions of $t_{L}, t_{R}$, and $\nu$. In particular, for $t_{L}<t_{R}$,

$$
\begin{array}{ll}
\lambda_{2}=\alpha \hat{t}+(1-\alpha) t_{L} ; & \lambda_{1}=\max \left\{\beta \hat{t}+(1-\beta) t_{L}, 0\right\} ; \\
\rho_{1}=\alpha \hat{t}+(1-\alpha) t_{R} ; & \rho_{2}=\min \left\{\beta \hat{t}+(1-\beta) t_{R}, 1\right\} ;
\end{array}
$$

where, $\alpha=\frac{g^{\prime}\left(\chi\left(t_{L}, t_{R}\right)\right)\left(t_{R}-t_{L}\right)}{g^{\prime}\left(\chi\left(t_{L}, t_{R}\right)\right)\left(t_{R}-t_{L}\right)+2}, \beta=\frac{g^{\prime}\left(\chi\left(t_{L}, t_{R}\right)\right)\left(t_{R}-t_{L}\right)}{g^{\prime}\left(\chi\left(t_{L}, t_{R}\right)\right)\left(t_{R}-t_{L}\right)-2}$, and $\hat{t}=g(\nu) t_{L}+(1-g(\nu)) t_{R}$.
} 


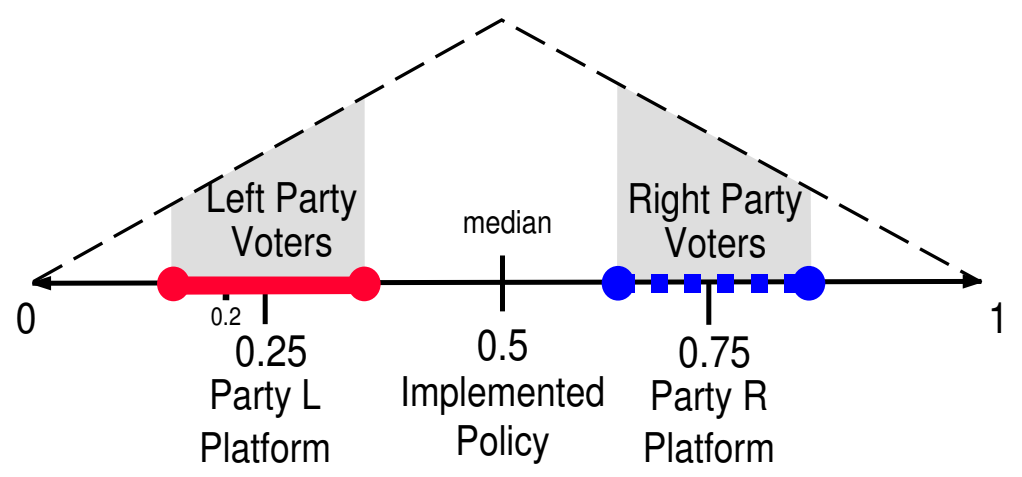

(a) Symmetric distribution of the electorate.

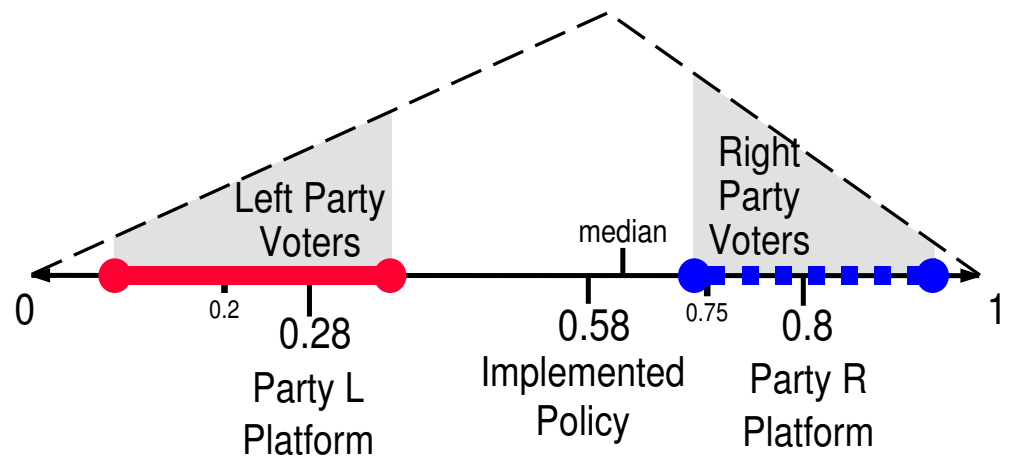

(b) Right-skewed distribution of the electorate.

Figure 5: Typical Equilibria for Symmetric and Asymmetric Distributions of the Electorate.

(iii) One party wins. Parties may receive different shares of the vote cast. Due to the shift to the right of the electorate in the asymmetric case, the right party obtains $55 \%$ of the votes and wins the election. The left party gets $45 \%$ of the vote and becomes the opposition. Since losers do not disappear after the elections, party $L$ still have a reason to participate in the election. This is in contrast with all dominant models, with or without uncertainty, that need a positive probability of winning for a party to enter the elections. ${ }^{15}$

(iv) Substantial turnout rates obtain despite the positive voting cost. Observe that all voters bear a cost of voting from supporting a party via a platform far form their ideal policy. Nevertheless, turnout rates are "high" (around $37 \%$ and $40 \%$, respectively).

(v) Voters locate in "bands" around the parties' platforms. That is, each

\footnotetext{
${ }^{15}$ Naturally, in the symmetric case both parties receive $50 \%$ of the vote.
} 
party finds its constituency among those who have a similar ideal policies.

(vi) Abstention results from alienation and from indifference. Because preferences are concave, citizens with extreme views benefit the most from shifts in the implemented policy. However, their preferred party's platform is so far from their ideal policy that they decide not to vote. Hence they abstain due to alienation (i.e. large costs of voting). On the other hand, voters close to the implemented policy are basically indifferent to small changes in the policy. They abstain due to small benefits of voting. Observe that if we removed alienation by letting all citizens face the same constant cost of voting, extreme voters would vote while moderates may abstain.

\section{Costless Voting}

In this section, we assume voting is costless. One could think of this restricted version of the general model as a comparative statics exercise: we recreate the standard political competition model with certainty, substituting the implemented policy function for the winner-takes-all assumption. That is, in our model the opposition party does not disappear from the analysis after the election. Thus, by maintaining the other basic assumptions (including certainty about voting behavior), we can understand the effects of giving a role to the opposition in explaining parties' and voters' behavior. The "exercise" becomes even more interesting when we discover that perturbing the winner-takes-all assumption produces diametrical results: full divergence of platforms and incentives for all citizens to vote. ${ }^{16}$ This is in contrast with the winner-takes-all case where platforms fully converge and no citizen has an incentive to vote because both parties announce the same platform and hence no vote is pivotal.

For the rest of this section we assume no costs of voting. ${ }^{17}$

Assumption 2 (A2) For any $\tau, \eta(t ; \tau)=0$ for all $t \in T$.

The main conclusions of this section are collected in Theorem 4.1: (i) the equilibrium is unique and (ii) there are only two types of equilibria in which either one party manages to implement its ideal policy, or, more interestingly, parties radicalize and announce extreme platforms.

Lemma 4.1 Let A1 and A2 hold. Then:

\footnotetext{
${ }^{16}$ To be precise, except for a zero-measure set of voters with ideal policy equal to the implemented policy, the rest of the electorate prefers voting to abstaining.

${ }^{17}$ Alternatively, we could allow for a 'civil duty' benefit and assume a positive reward from voting $(\eta(t ; \tau)=k<0)$ without altering the results.
} 
(i) Given $t_{L}, t_{R} \in T$ and $\nu \in[0,1]$, everybody votes.

Moreover, for $t_{L}<t_{R}$, everybody to the left of $\hat{t}\left(t_{L}, t_{R}, \nu\right)$ votes for $L$ while everybody to the right of $\hat{t}\left(t_{L}, t_{R}, \nu\right)$ votes for $R$.

That is, $L\left(t_{L}, t_{R}, \nu\right)=\left[\underline{t}, \hat{t}\left(t_{L}, t_{R}, \nu\right)\right)$, and $R\left(t_{L}, t_{R}, \nu\right)=\left(\hat{t}\left(t_{L}, t_{R}, \nu\right), \bar{t}\right]$.

(ii) $\chi\left(t_{L}, t_{R}\right)$ is non-empty and single-valued for all $\left(t_{L}, t_{R}\right) \in T^{2}$.

(iii) $\chi$ is continuous, except possibly at $t_{L}=t_{R}$.

The proofs of the lemmas are relegated to Appendix B.

Lemma 4.1 shows that voters vote for the party that represent their interests: everybody with an ideal policy to the left of the expected implemented policy votes for the Left party, while those citizens with a preferred policy to the right of the implemented policy vote for the Right party. Hence everybody votes. Moreover, there exist a unique consistent vote for each pair of platforms and the voting outcome function is continuous. Because $\chi$ is a function, we can project the political game on the policy space. Thus we are able to formulate the implemented policy and the utility functions of parties in reduced form. Let $\tilde{t}: T^{2} \rightarrow T$ be defined by

$$
\tilde{t}\left(t_{L}, t_{R}\right)=\hat{t}\left(t_{L}, t_{R}, \chi\left(t_{L}, t_{R}\right)\right) .
$$

For $J=L, R$, let $\tilde{\pi}_{J}: T \times T \rightarrow \mathbb{R}$ be defined by

$$
\tilde{\pi}_{J}\left(t_{L}, t_{R}\right)=\pi_{J}\left(\tilde{t}\left(t_{L}, t_{R}\right)\right) .
$$

It follows (Lemma 4.2) that the implemented policy function and the utility of parties are also continuous on $T \times T$.

Lemma 4.2 The functions $\tilde{t}$ and $\tilde{\pi}_{J}$ are continuous on $T^{2}$.

Define the best-response correspondence of party $J=L, R, B R_{J}: T \rightrightarrows T$, that assigns to each alternative the set of utility maximizers. That is,

$$
\begin{array}{l|l}
B R_{L}\left(t_{R}\right)=\arg \max \left\{\tilde{\pi}_{L}\left(t, t_{R}\right)\right. & \mid t \in T\} \\
B R_{R}\left(t_{L}\right)=\arg \max \left\{\tilde{\pi}_{R}\left(t_{L}, t\right)\right. & \mid t \in T\} .
\end{array}
$$

The following lemma shows that the best response for each party is to radicalize, unless it can ensure the implementation of its ideal policy.

Lemma 4.3 Let A1 and A2 hold.

(i) The best-response correspondence of party $J, B R_{J}$, is single-valued and continuous, $J=L, R$. 
(ii) Given $t_{R} \geq \tau_{L}$, either $L$ can implement its ideal policy, or its best response is $\underline{t}$. That is, either there exists $t_{L}^{o}$ such that $\tilde{t}\left(t_{L}^{o}, t_{R}\right)=\tau_{L}$, or $B R_{L}\left(t_{R}\right)=\{\underline{t}\}$.

(iii) Given $t_{L} \leq \tau_{R}$, either $R$ can implement its ideal policy, or its best response is $\bar{t}$. That is, either there exists $t_{R}^{o}$ such that $\tilde{t}\left(t_{L}, t_{R}^{o}\right)=\tau_{R}$, or $B R_{R}\left(t_{L}\right)=\{\bar{t}\}$.

It follows from the previous lemmas that there is a unique political equilibrium which can take only two possible forms: either one party implements its ideal policy or parties radicalize by announcing extreme platforms.

Theorem 4.1 Let A1 and A2 hold. Then:

(i) a political equilibrium exists;

(ii) the equilibrium is unique; and

(iii) either platforms are polarized at equilibrium (i.e., $\left.\left(t_{L}^{*}, t_{R}^{*}\right)=(\underline{t}, \bar{t})\right)$, or one of the parties can implement its ideal policy $\left(i . e . \tilde{t}\left(t_{L}^{*}, t_{R}^{*}\right)=\tau_{J}\right.$, for some $J=L, R$.)

Proof.

(i) Existence. Let $B R: T^{2} \rightarrow T^{2}$ defined by $B R\left(t_{L}, t_{R}\right)=\left(B R_{L}\left(t_{R}\right), B R_{R}\left(t_{L}\right)\right)$. From Lemma $4.3, B R$ is a continuous function. Thus, by Brouwer's fixed point theorem, there exists $\left(t_{L}^{*}, t_{R}^{*}\right) \in T^{2}$ such that $B R\left(t_{L}^{*}, t_{R}^{*}\right)=\left(t_{L}^{*}, t_{R}^{*}\right)$. It follows that $\left(t_{L}^{*}, t_{R}^{*}\right)$ is an equilibrium.

(iii) Let $\left(t_{L}^{*}, t_{R}^{*}\right)$ be an equilibrium and suppose that $\left(t_{L}^{*}, t_{R}^{*}\right) \neq(\underline{t}, \bar{t})$. If $t_{L}^{*} \neq \underline{t}$, then $\tilde{t}\left(t_{L}^{*}, t_{R}^{*}\right)=\tau_{L}$ (Lemma 4.3). If $t_{L}^{*}=\underline{t}$, then $t_{R}^{*} \neq \bar{t}$ and $\tilde{t}\left(t_{L}^{*}, t_{R}^{*}\right)=\tau_{R}$ (Lemma 4.3 , once more).

(ii) Uniqueness. Suppose that $\left(t_{L}^{1}, t_{R}^{1}\right)$ and $\left(t_{L}^{2}, t_{R}^{2}\right)$ are two different equilibria. From (iii), at least one of them is not $(\underline{t}, \bar{t})$ and it implements the ideal policy of one party. Without loss of generality, take $\left(t_{L}^{1}, t_{R}^{1}\right) \neq(\underline{t}, \bar{t})$, and suppose that $\tilde{t}\left(t_{L}^{1}, t_{R}^{1}\right)=\tau_{L}$ (nothing would change had we taken $\tau_{R}$ instead).

Because $\tau_{L}$ is implemented, $B R_{R}\left(t_{L}^{1}\right)=\bar{t}$ (Lemma 4.3). That is, $\left(t_{L}^{1}, \bar{t}\right)$ is an equilibrium and, therefore, $t_{L}^{1}=B R_{L}(\bar{t})$. It follows that $\left(t_{L}^{2}, t_{R}^{2}\right) \neq(\underline{t}, \bar{t})$ as well, since $B R_{L}$ is a function and $\left(t_{L}^{2}, t_{R}^{2}\right) \neq\left(t_{L}^{1}, \bar{t}\right)$. Thus it must be that $\tilde{t}\left(t_{L}^{2}, t_{R}^{2}\right)=\tau_{R}$ and $t_{L}^{2}=\underline{t}$, by a similar reasoning as above.

Therefore, we have shown that $\tilde{t}\left(t_{L}^{1}, t_{R}^{1}\right)=\tau_{L}, \tilde{t}\left(t_{L}^{2}, t_{R}^{2}\right)=\tau_{R}, t_{R}^{1}=\bar{t}$ and $t_{L}^{2}=\underline{t}$. ¿From Lemma 4.1, $\chi\left(t_{L}^{1}, t_{R}^{1}\right)=F\left(\tau_{L}\right)$ and $\chi\left(t_{L}^{2}, t_{R}^{2}\right)=F\left(\tau_{R}\right)$. Then, using the definition of $\tilde{t}$,

$$
\begin{aligned}
\tau_{L} & =\tilde{t}\left(t_{L}^{1}, \bar{t}\right)=g\left(F\left(\tau_{L}\right)\right) t_{L}^{1}+\left(1-g\left(F\left(\tau_{L}\right)\right)\right) \bar{t}, \text { and } \\
\tau_{R}=\tilde{t}\left(\underline{t}, t_{R}^{2}\right) & =g\left(F\left(\tau_{R}\right)\right) \underline{t}+\left(1-g\left(F\left(\tau_{R}\right)\right)\right) t_{R}^{2} .
\end{aligned}
$$


Solving for $t_{L}^{1}$ and $t_{R}^{2}$, we obtain

$$
\begin{aligned}
t_{L}^{1} & =\frac{\tau_{L}-\left(1-g\left(F\left(\tau_{L}\right)\right)\right) \bar{t}}{g\left(F\left(\tau_{L}\right)\right)} \geq \underline{t}, \\
t_{R}^{2} & =\frac{\tau_{R}-g\left(F\left(\tau_{R}\right)\right) \underline{t}}{1-g\left(F\left(\tau_{R}\right)\right)} \leq \bar{t} .
\end{aligned}
$$

Rearranging terms, we obtain

$$
\begin{aligned}
\tau_{L} & \geq g\left(F\left(\tau_{L}\right)\right) \underline{t}+\left(1-g\left(F\left(\tau_{L}\right)\right)\right) \bar{t} \\
\tau_{R} & \leq g\left(F\left(\tau_{R}\right)\right) \underline{t}+\left(1-g\left(F\left(\tau_{R}\right)\right)\right) \bar{t} .
\end{aligned}
$$

But the right hand side of the first inequality is larger than the right hand side of the second inequality (since $g$ is a non-decreasing function) contradicting $\tau_{L}<\tau_{R}$.

Thus, in a very general setting, the standard convergence tendency of platforms disappears whenever the opposition party interferes in policymaking. Therefore, platform convergence cannot be explained as the result of party competition, but as a consequence of excluding the opposition from any post-electoral process. That is, parties converge in their platforms because they disappear if they lose the election.

\section{$5 \quad$ Political Equilibrium with Costly Voting}

In the previous section we showed that if the opposition does not disappear after the election, parties polarize their positions. ${ }^{18}$ Recall that an almost winner-takesall implemented policy function, like the one in Figure 2(a), would suffice to make parties announce radical platforms.

As argued in the introduction, we cannot ignore that platforms and policies will not generically coincide and hence that voters may consider both when deciding whether to vote and for whom. Therefore, we incorporate a cost that depends on the positions defended by the parties. Despite this cost, a sizable share of the electorate will still vote. We show that parties do not radicalize their platforms anymore, although a tendency towards divergence is still present. The intuition is simple. Voters care about the platform they support, and may feel alienated by a party that locates itself too far from their ideal policy. If the electorate concentrates in the middle (i.e., if it holds fairly moderated views), a party will alienate the core of its constituency by announcing an extreme platform. That is, it is not that the

\footnotetext{
${ }^{18}$ This centrifugal force over parties' platforms distinguishes our results from other explanations of platform differentiation, where platforms are subject to a centripetal force but they do not fully converge.
} 
divergence tendency does disappear when we consider costly voting, instead it finds an opposing force that diminishes its effect.

Thus, voters will consider the electoral platforms when casting their votes (or abstaining). In this sense voting is costly. Let the cost of voting be an increasing and weakly convex function of the distance between the platform of the party and the preferred policy of the voter:

$$
\eta(t ; \tau)=\zeta(|t-\tau|), \text { with } \zeta^{\prime}>0, \zeta^{\prime \prime} \geq 0, \text { and } \zeta(0)=0 .
$$

We shall assume through this section that policy formation follows a proportional representation system (as defined in Persson and Tabellini, 2000; Lizzeri and Persico, 2001; Gerber and Ortuño-Ortín, 1998; Ortuño-Ortín, 1997), namely $\hat{t}\left(t_{L}, t_{R}, \nu\right)=$ $\nu t_{L}+(1-\nu) t_{R}$, for all $\left(t_{L}, t_{R}, \nu\right) .{ }^{19}$ Let the policy space be the unit interval, $T=[0,1]$. In addition, we shall assume that the benefit of voting for a party is a weakly concave function of $\tau$. That is, $\frac{\partial^{2} w(L, \tau)}{\partial \tau^{2}} \leq 0 .^{20}$ In other words, since voters further from the implemented policy are more sensitive to changes in the policy (this follows directly from the concavity of the utility function), we shall assume that this sensitivity increases at a decreasing rate. ${ }^{21}$

A first result shows each party's constituency is concentrated in an interval around the platform.

Theorem 5.1 Given $t_{L}, t_{R}$, and $\nu$, with $t_{L} \neq t_{R}$ and $\nu \in(0,1)$, the voters of party $L$ concentrate in an interval around $t_{L}$, while the voters of party $R$ concentrate in an interval around $t_{R}$. That is,

$$
\begin{aligned}
& L\left(t_{L}, t_{R}, \nu\right)=\left[\lambda_{1}\left(t_{L}, t_{R}, \nu\right), \lambda_{2}\left(t_{L}, t_{R}, \nu\right)\right] \ni t_{L}, \text { and } \\
& R\left(t_{L}, t_{R}, \nu\right)=\left[\rho_{1}\left(t_{L}, t_{R}, \nu\right), \rho_{2}\left(t_{L}, t_{R}, \nu\right)\right] \ni t_{R} .
\end{aligned}
$$

Proof. Take $t_{L}, t_{R} \in T$ and $\nu \in(0,1)$. Let $t_{L}<t_{R}$ (the proof can be easily replicated for $\left.t_{L}>t_{R}\right)$. Write $\tilde{w}(s ; \tau)=w\left(s ; t_{L}, t_{R}, \nu, \tau\right), \tilde{\eta}(s ; \tau)=\hat{\eta}\left(s ; t_{L}, t_{R}, \tau\right)$, $\tilde{v}(s ; \tau)=\tilde{w}(s ; \tau)-\tilde{\eta}(s ; \tau)$, and $t^{*}=\hat{t}\left(t_{L}, t_{R}, \nu\right)$.

\footnotetext{
${ }^{19}$ Proportional representation is taken for the sake of presentation. For instance, section 3 does present an example with a non-proportional weight function.

${ }^{20}$ For instance, any concave utility as a function of the distance between the policy and the ideal point of the agent would satisfy this condition.

${ }^{21}$ As it will become evident through the analysis, the weakly concavity of the benefit of voting is equivalent to the single-crossing property, commonly used in voting models, since it implies that voters for each party form a connected set.

The need for these specifications derives from the fact the possibility of abstention engenders equilibrium existence problems even in the standard winner-takes-all system (Llavador, 2000). Although it could be relaxed in many ways without affecting the results, we choose the current specification for the shake of simplicity.
} 
First, from Lemma 4.1, a citizen to the left(right) of $t^{*}$ either votes for party $L$ (party $R$ ) or abstain. Second, observe that $v\left(L, t^{*}\right)<0$ (since $w\left(L, t^{*}\right)=0<$ $\left.\eta\left(t_{L}, t^{*}\right)\right)$, while $v\left(L, t_{L}\right)>0\left(w\left(L, t_{L}\right)>0=\eta\left(t_{L}, t_{L}\right)\right)$. Finally, because $w$ is concave in $\tau, \eta$ is convex in $\tau$ and $v=w-v$, it follows that $v$ is concave in $\tau$. Thus, the upper-contour sets of $v$ are convex sets. In particular, $V_{L}\left(t_{L}, t_{R}, \nu\right)=$ $\tau \in[0,1]: v(L, \tau) \geq 0$ is a convex set which contains $t_{L}\left(v\left(L, t_{L}\right)>0\right)$.

A similar argument applies for $V_{R}$.

Furthermore, there is always a positive mass of citizens who decide to vote:

Corollary 5.1 For any $t_{L}, t_{R}$, and $\nu$ with $t_{L} \neq t_{R}$, there exists always a positive mass of citizens who vote. That is,

$$
V_{L}\left(t_{L}, t_{R}, \nu\right)+V_{R}\left(t_{L}, t_{R}, \nu\right)>0 \quad \forall\left(t_{L}, t_{R}, \nu\right) \text { with } t_{L} \neq t_{R}
$$

Proof. If $0<\nu<1$, it follows directly from Theorem 5.1. If $\nu=0$ then $V_{L}>0\left(\right.$ since $\left.t^{*}>t_{L}\right)$, while if $\nu=1, V_{R}>0\left(t^{*}<t_{R}\right)$.

The following technical lemmas are used for the existence and characterization of equilibria. First, we show that the share of the vote cast for a party is a continuous, monotone function. It will follow that there exists a unique consistent voting outcome associated to each pair of platforms.

Lemma 5.1 Let $t_{L} \neq t_{R}$. Then $V_{L}$ and $V_{R}$ are continuous functions, $V_{L}$ is nonincreasing in $\nu$, and $V_{R}$ is non-decreasing in $\nu$.

Lemma 5.2 The consistent voting outcome correspondence $\chi$ is non-empty, singlevalued, and continuous for all $t_{L} \neq t_{R}$.

When both parties propose identical platforms, the implemented policy is unmistakably the common platform, independent of the voting outcome. Therefore, using Lemma 5.2 we can write the implemented policy as:

$$
\tilde{t}\left(t_{L}, t_{R}\right)= \begin{cases}\hat{t}\left(t_{L}, t_{R}, \chi\left(t_{L}, t_{R}\right)\right) & \text { if } t_{L} \neq t_{R}, \\ t & \text { if } t_{L}=t_{R}=t .\end{cases}
$$

Consider first a symmetrically distributed electorate. Not surprisingly, equilibria present symmetry features: parties propose symmetric policies, the vote is equally split, and the implemented policy is the median policy, which, in this case, coincides with the mean. More importantly, while parties propose differentiated platforms, they do not (necessarily) take extreme positions. ${ }^{22}$ For instance, a sufficiently high voting cost and a fairly concentrated distribution of the electorate would drive parties to moderate their proposals.

\footnotetext{
${ }^{22}$ We might argue that symmetry should facilitate location at the extremes. Therefore, finding non-extreme platforms for symmetrically distributed populations strengthen our argument.
} 
Theorem 5.2 Let the electorate be distributed symmetrically around $\frac{1}{2}: f(x)=$ $f(1-x)$. If $\tau_{L} \leq \frac{1}{2} \leq \tau_{R}$ and $\tau_{L} \neq \tau_{R}$, then:

(i) there exists a political equilibrium $\left(t_{L}^{*}, t_{R}^{*}, \nu^{*}\right)$, with $t_{L}^{*}=\frac{1}{2}-k$ and $t_{R}^{*}=\frac{1}{2}+k$ for some $k \in[0,1 / 2)$;

(ii) at any equilibria, the implemented policy is $t^{*}=\frac{1}{2}$, and both parties receive the same share of votes, $\nu^{*}=\frac{1}{2}$.

Proof. We proceed in steps.

- First we construct an auxiliary zero-sum game and show that there always exists an equilibrium for this game.

Consider the game $\Gamma=\left(2, T, \Pi_{L}, \Pi_{R}\right)$, where the payoffs of the parties are $\Pi_{L}\left(t_{L}, t_{R}\right)=-\tilde{t}\left(t_{L}, t_{R}\right)$ and $\Pi_{R}\left(t_{L}, t_{R}\right)=\tilde{t}\left(t_{L}, t_{R}\right)$, respectively. This is a two-party zero-sum game. Define the security level for each party as

$$
\begin{aligned}
\tilde{t}_{R}^{0} & =\max _{t_{R}} \min _{t_{L}} \tilde{t}\left(t_{L}, t_{R}\right), \text { and } \\
\tilde{t}_{L}^{0} & =\min _{t_{L}} \max _{t_{R}} \tilde{t}\left(t_{L}, t_{R}\right) .
\end{aligned}
$$

Because party $R$ can surely implement at least $\tilde{t}_{R}^{o}$ and party $L$ can keep the policy from being more than $\tilde{t}_{L}^{o}$, then it must be that $\tilde{t}_{R}^{0} \leq \tilde{t}_{L}^{0}$. On the other hand, a party can always impose a policy $t^{*}=\frac{1}{2}$ by announcing a symmetric platform to its opponent's. It follows that $\tilde{t}_{L}^{0} \leq \frac{1}{2}$ and $\tilde{t}_{R}^{0} \geq \frac{1}{2}$. Therefore, $\tilde{t}_{L}^{0}=\tilde{t}_{R}^{0}=\frac{1}{2}$. Thus, an equilibrium of $\Gamma$ exits and the implemented policy is $\frac{1}{2}$.

- Secondly, we show that if $\left(t_{L}^{*}, t_{R}^{*}\right)$ is an equilibrium of $\Gamma$ and $\nu^{*}=\Xi\left(t_{L}^{*}, t_{R}^{*}\right)$, then $\left(t_{L}^{*}, t_{R}^{*}, \nu^{*}\right)$ is a political equilibrium, proving point (i) of the theorem. Because $\left(t_{L}^{*}, t_{R}^{*}\right)$ is an equilibrium of $\Phi, \tilde{t}\left(t_{L}^{*}, t\right) \leq \tilde{t}\left(t_{L}^{*}, t_{R}^{*}\right) \leq \tilde{t}\left(t, t_{R}^{*}\right)$ for all $t$, i.e., party $L$ cannot lower the implemented policy, neither can party $R$ increase it. Recall that $\tau_{L}<\frac{1}{2}=\tilde{t}\left(t_{L}^{*}, t_{R}^{*}\right)$ an $\pi_{L}$ is single-peaked, it follows then that $t_{L}^{*}$ is a best response for $L$ to $t_{R}^{*}$. Viceversa $t_{R}^{*}$ is also a best response for $R$ to $t_{L}^{*}$. Thus, $\left(t_{L}^{*}, t_{R}^{*}, \nu^{*}\right)$ is a political equilibrium.

- Finally, observe that party $R$ will never propose a policy $t_{R}<\tau_{L}$, for it is a strictly dominated strategy. Similarly, party $L$ will never propose $t_{L}>\tau_{R}$. Thus, after eliminating strictly dominated strategies, the political game is a strictly competitive game, and hence all equilibria yield the same payoffs for the parties: $t^{*}=\frac{1}{2}$. 


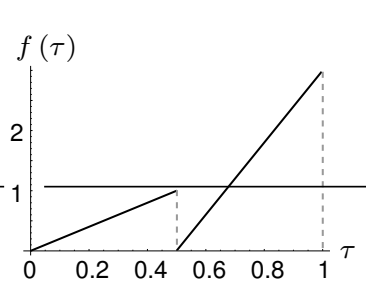

(a) Density function.

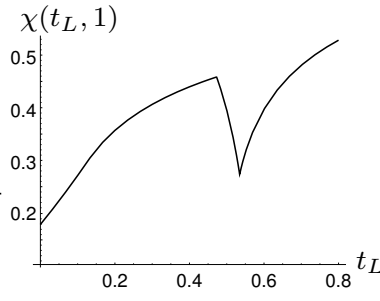

(b) $\chi$ is not quasi-concave in $t_{L}$ for $t_{R}=1$.

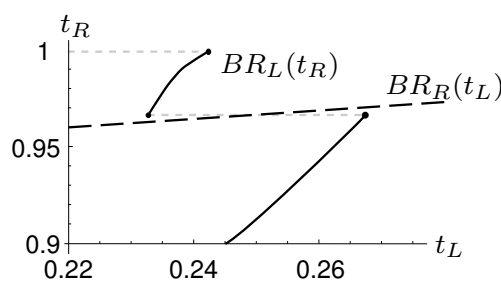

(c) An equilibrium does not exist: the reaction curves do not intersect.

Figure 6: Example of non-existence of equilibrium for very asymmetric distributions. The density function is bi-triangular and $\eta(t ; \tau)=-5|t-\tau|$.

The results found under symmetry extend to more general distributions of the electorate. However, for highly asymmetric distributions (mainly displaying more than one peak), one may construct examples where an equilibrium in pure strategies fails to exist ${ }^{23}$. Figure 6 illustrates such a $\operatorname{case}^{24}$ (details available from the author.) However, these examples are not common and involve very strong asymmetries. We choose to postulate a sufficient condition for the existence of equilibria. In particular, we take $\chi$ to be log-concave, a premise also known in the literature as decreasing hazard rate. ${ }^{25}$ Figure $6(\mathrm{~b})$ shows that this condition is violated in the non-existence example.

Theorem 5.3 Let $\chi$ be log-concave in $t_{L}$ and let $1-\chi$ be log-concave in $t_{R}$. Then:

(i) a political equilibrium exists,

(ii) at the equilibrium parties propose differentiated but not necessarily extreme policies, and

(iii) the implemented policy is the same in all equilibria.

Proof. We follow the steps of the symmetric case.

\footnotetext{
${ }^{23}$ However, there exits always an equilibrium in mixed strategies (see Fudenberg and Tirole (Theorem 1.3 in 1991).)

${ }^{24} \mathrm{As}$ mentioned before, equilibrium existence is also an issue in the classic winner-takes-all model with abstention and multi-peaked distributions of the electorate (Llavador, 2000).

${ }^{25}$ Ideally, such assumptions would be imposed on the primitives of the model $(g, F, u$, and $\eta)$, but because $\chi$ is implicitly derived from a complex computation, this is not an easy task. Instead, we follow (Roemer, 1997, p.492) who, in a similar context, also requires the log-concavity of a compound function.
} 
- First, we construct a zero-sum game $\Gamma$ and prove that an equilibrium always exists in this game.

Consider the two-party, zero-sum game $\Gamma=\left(T, \Pi_{L}, \Pi_{R}\right)$, with payoff functions $\Pi_{L}\left(t_{L}, t_{R}\right)=-\hat{t}\left(t_{L}, t_{R}, \chi\left(t_{L}, t_{R}\right)\right)$ and $\Pi_{R}\left(t_{L}, t_{R}\right)=\hat{t}\left(t_{L}, t_{R}, \chi\left(t_{L}, t_{R}\right)\right)$, respectively. Use the implemented policy function to write:

$$
\begin{aligned}
& \Pi_{L}\left(t_{L}, t_{R}\right)=-t_{R}+\chi\left(t_{L}, t_{R}\right)\left(t_{R}-t_{L}\right), \text { and } \\
& \Pi_{R}\left(t_{L}, t_{R}\right)=t_{L}+\left(1-\chi\left(t_{L}, t_{R}\right)\right)\left(t_{R}-t_{L}\right) .
\end{aligned}
$$

Therefore, maximizing $\Pi_{L}$ (with respect to $t_{L}$ ) and $\Pi_{R}$ (with respect to $t_{R}$ ) is equivalent to maximizing

$$
\begin{aligned}
& \kappa_{L}\left(t_{L}, t_{R}\right) \equiv \chi\left(t_{L}, t_{R}\right)\left(t_{R}-t_{L}\right) \text { and } \\
& \kappa_{R}\left(t_{L}, t_{R}\right) \equiv\left(1-\chi\left(t_{L}, t_{R}\right)\right)\left(t_{R}-t_{L}\right) .
\end{aligned}
$$

Next we show that the log-concavity of $\chi$ implies the quasi-concavity of $\kappa_{L}$. The function $\kappa_{L}$ is quasi-concave in $t_{L}$ if $\frac{\partial^{2} \kappa_{L}}{\partial t_{L}^{2}}\left(t_{L}, t_{R}\right) \leq 0$ for all $t_{L}$ such that $\frac{\partial \kappa_{L}}{\partial t_{L}}\left(t_{L}, t_{R}\right)=0$. Write $\chi_{L}=\frac{\partial \chi}{\partial t_{L}}$ and $\chi_{L L}=\frac{\partial^{2} \chi}{\partial t_{L}^{2}}$. We compute:

$$
\begin{aligned}
\frac{\partial \kappa_{L}}{\partial t_{L}}\left(t_{L}, t_{R}\right) & =\chi_{L}\left(t_{L}, t_{R}\right) \cdot\left(t_{R}-t_{L}\right)-\chi\left(t_{L}, t_{R}\right), \text { and } \\
\frac{\partial^{2} \kappa_{L}}{\partial t_{L}^{2}}\left(t_{L}, t_{R}\right) & =\chi_{L L}\left(t_{L}, t_{R}\right) \cdot\left(t_{R}-t_{L}\right)-2 \cdot \chi_{L}\left(t_{L}, t_{R}\right) .
\end{aligned}
$$

Let $t_{L} \in T$ such that $\frac{\partial \kappa_{L}}{\partial t_{L}}=0$, then from (5.3) we get $\left(t_{R}-t_{L}\right)=\frac{\chi\left(t_{L}, t_{R}\right)}{\chi_{L}\left(t_{L}, t_{R}\right)}$ and, substituting into (5.4), we obtain (all functions are evaluated at $\left(t_{L}, t_{R}\right)$ ):

$$
\left.\frac{\partial^{2} \kappa_{L}}{\partial t_{L}^{2}}\right|_{\frac{\partial \kappa_{L}}{\partial t_{L}}=0}=\left.\chi_{L L}\right|_{\frac{\partial \kappa_{L}}{\partial t_{L}}=0} \cdot \frac{\chi}{\left.\chi_{L}\right|_{\frac{\partial \kappa_{L}}{\partial t_{L}}=0}}-\left.2 \cdot \chi_{L}\right|_{\frac{\partial \kappa_{L}}{\partial t_{L}}=0} .
$$

Thus,

$$
\left.\frac{\partial^{2} \kappa_{L}}{\partial t_{L}^{2}}\right|_{\frac{\partial \kappa_{L}}{\partial t_{L}}=0} \geq\left. 0 \Leftrightarrow \chi_{L L}\right|_{\frac{\partial \kappa_{L}}{\partial t_{L}}=0} \cdot \chi-2 \cdot\left(\left.\chi_{L}\right|_{\frac{\partial \kappa_{L}}{\partial t_{L}}=0}\right)^{2} \leq 0
$$

since $\left.\chi_{L}\right|_{\frac{\partial \kappa_{L}}{\partial t_{L}}=0} \equiv \frac{\chi}{t_{R}-t_{L}}>0$.

The log-concavity of $\chi$ implies that $\chi_{L L} \cdot \chi-\left(\chi_{L}\right)^{2} \leq 0$. It follows from (5.5) that $\kappa_{L}$ is quasi-concave. A symmetric argument shows that the log-concavity of $(1-\chi)$ implies that $\kappa_{R}$ is quasi-concave in $t_{R}$. 
Let $\mathrm{BR}_{L}\left(t_{R}\right)$ be the set of maximizers of $\Pi_{L}\left(t_{L}, t_{R}\right)$ for a given $t_{R}$ (i.e., the best response of $L$ to $t_{R}$ ). Since $\kappa_{L}$ is quasi-concave, $\mathrm{BR}_{L}$ is convex-valued. From the Theorem of the Maximum, $\mathrm{BR}_{L}$ is non-empty and upper hemi-continuous. Similarly, $\mathrm{BR}_{R}$ is also non-empty, convex-valued and upper hemi-continuous. Then, the correspondence $\mathrm{BR}: T^{2} \rightarrow T^{2}: \mathrm{BR}\left(t_{L}, t_{R}\right)=\left(\mathrm{BR}_{L}\left(t_{R}\right), \mathrm{BR}_{R}\left(t_{L}\right)\right)$ is non-empty, convex-valued and upper hemi-continuous. By Kakutani's fixed point theorem there exists a $\left(t_{L}^{*}, t_{R}^{*}\right) \in \mathrm{BR}\left(t_{L}^{*}, t_{R}^{*}\right)$. Therefore, $\left(t_{L}^{*}, t_{R}^{*}\right)$ is an equilibrium of $\Gamma$.

- Secondly, let $\left(t_{L}^{*}, t_{R}^{*}\right)$ be an equilibrium of the zero-sum game $\Gamma$ and let $t^{*}=$ $\tilde{t}\left(t_{L}^{*}, t_{R}^{*}\right)$ be the equilibrium policy. If $t^{*}$ locates between the ideal policies of the parties $\left(\tau_{L}<t^{*}<\tau_{R}\right)$, then these policies constitute an equilibrium of the political game.

If $\tau_{L} \leq t^{*} \leq \tau_{R}$, then neither party can pull the implemented policy towards its ideal point given the platform announced by the other party. Hence, $\left(t_{L}^{*}, t_{R}^{*}\right)$ is a political equilibrium.

- Thirdly, if $t^{*}$ is more extreme than a party's bliss point $\left(t^{*}<\tau_{L}\right.$ or $\left.t^{*}>\tau_{R}\right)$, then there exists a political equilibrium where that party is able to implement its ideal policy.

Take, for example, $t^{*}<\tau_{L}$. Since $\Gamma$ is a zero-sum game, it follows that $L$ can secure a policy as to the left as $t^{*}$. Because $\tau_{L}>t^{*}, t^{*}$ represents a lower payoff for $L$ in the zero-sum game, and party $L$ can definitely secure $\tau_{L}$. Thus, if $t^{*}<\tau_{L}$, there exists a political equilibrium where the implemented policy is $\tau_{L}$. Similarly, if $t^{*}>\tau_{R}$, there exists a political equilibrium with $\tau_{R}$ as the implemented policy.

- It follows from the previous two points that an equilibrium of the political game always exists. Part (i) is proved.

- Party $R$ will never propose a policy $t_{R}<\tau_{L}$, nor party $L$ will ever propose $t_{L}>$ $\tau_{R}$. Thus, after eliminating these strictly dominated strategies, the political game is a strictly competitive game and hence the implemented policy is the same in all equilibria, proving part (iii)

- Finally, we prove part (ii). Observe that one can never have an equilibrium where both parties propose the same policy. At least one party can always pull the policy a bit closer to its ideal point by differentiating its platform and capturing a small fraction of the vote. As the example in Section 3 shows, platforms may not be extreme at equilibrium. 
Summarizing, we provide sufficient conditions for equilibrium existence. At equilibrium, voters concentrate around the platforms of the parties and, despite positive voting costs, high turnout rates obtain. Abstention occurs among both citizens with extreme and moderate views and can be explained in terms of alienation and indifference. Parties announce differentiated but non-radical platforms, still they may choose platforms that are more extreme than their ideal policy. The implemented policy tends to be moderated and unrelated to the ideal policy of the median voter. Thus our results are consistent with the observation of polarized platforms and moderate policies.

\section{Concluding Remarks}

This paper enriches the theoretical framework of political competition to allow for non-trivial policy-setting processes and for sophisticated voters. We let the implemented policy depend on the electoral platforms and the margin of victory. Voters may vote for one of the candidates or abstain. Since platforms and implemented policies may differ, voting for the preferred candidate is not always a dominant strategy. Thus, we cannot ignore that voters may consider both the electoral platform and the effect on the implemented policy when deciding whether to vote and for whom.

First, we show that in the predominant framework of costless voting, a divergence tendency drives parties to take extreme positions. This centrifugal force differentiates our results from other explanations of party differentiation. The key assumption driving our explanation is that the opposition party does not disappear from the political arena after loosing the election. Rather, its platform and electoral support affect (to some extent) the implemented policy. Considering rational voters allows us to prove equilibrium existence for a generic specification of the implemented function and any distribution of the electorate.

Secondly, we introduce costly voting (voters care about supporting a party too alien to their political preferences). We provide sufficient conditions for equilibrium existence and show that equilibria are characterized by differentiated, yet not necessarily radical, platforms. ${ }^{26}$ Here, it is the risk of alienating one's core constituency what drives partial convergence in parties' platforms. High turnout rates obtain, even with positive costs of voting. Abstention occurs among voters with extreme as well as moderate views. The implemented policy tends to be moderated and unrelated to the ideal policy of the median voter. Thus our results are consistent with both the observation of polarized platforms and moderate policies, and the alienation and indifference causes of abstention.

\footnotetext{
${ }^{26}$ Nevertheless, platforms may be more extreme than parties' ideal policies.
} 
More importantly, the analysis evidences that the distribution of the electorate, and not only the (expected) location of a pivotal voter, matters in determining policy outcomes. Consider, for example, two countries identical on everything but on the distribution of their electorate. While country A's electorate follows a concentratedin-the-middle, unimodal distribution, the electorate of country B is fractured into two radical groups (its density function presents two peaks with the majority of the population located around them). Assume that they share the same median voter, or, if there is uncertainty, that the knowledge about the location of the median voter is the same in both countries. According to our model, parties in country A would move towards the center to avoid alienating the core of their constituency, while parties in country B will radicalize their positions, following the radicalization of the electorate. ${ }^{27}$ However, models based on the median voter are insensitive to the distribution of the population and would predict the same electoral outcome in both countries.

Summarizing our results, we should observe parties moving towards the 'middle' of the political spectrum when $(i)$ the mass of the electorate is concentrated around moderate views, and (ii) parties alienate the core of their constituencies if they radicalize their platforms. This is in contrast to the standard claims that parties tend to the middle because of competition, and that platforms should converge as the uncertainty level falls (ceteris paribus). In this sense, our model is consistent with the observation that electorate polarization and elite political divergence have moved in tandem for the past half century. It also sheds light on the radicalization of parties' positions in those regions, like Israel or the Basque Country, where the electorate is divided and polarized.

\section{Appendix}

\section{A A Limiting Argument for the Benefit of Voting}

In this appendix we present a justification for the benefit of voting -as described in expression 2.3- based on the impact of a citizen's vote on the implemented policy when the number of voters go to infinity.

First, for a given pair of announced platforms $\left(t_{L}, t_{R}\right)$, we write the utility of a voter as a function of her expected electoral outcome

$$
\mathbf{u}_{\tau}(\nu)=u\left(\hat{t}\left(t_{L}, t_{R}, \nu\right) ; \tau\right)
$$

\footnotetext{
${ }^{27}$ The implemented policy in country B may or may not be radical, depending on the relative weight of each group and on post-electoral policymaking process (the function $g$ in the model.)
} 
Consider a society with a finite number of voters $n$. Observe that depending on the value of $n$, it may not be 'possible' to expect an exact $\nu \%$ of the population voting for $L$. Therefore, given a $\nu \in(0,1)$, we need to define $\nu(n)$. We know we can write $\nu=\frac{p}{q}$ with $p, q \in \mathbb{N}$. (In particular, take $p, q$ such that $q$ is the minimum integer with $\nu=\frac{p}{q}$.) For each $n$ choose the largest feasible fraction of the vote less than or equal to $\nu$

$$
\nu(n)=\frac{\lfloor n \cdot \nu\rfloor}{n},
$$

where $\lfloor n \cdot \nu\rfloor$ gives the largest integer less than or equal to $n \cdot \nu .^{28}$

Now, we can calculate the benefit of voter $\tau$ from voting for $L$ when she expects $\nu(n)$ as

$$
\mathbf{u}_{\tau}\left(\nu(n)+\frac{1}{n}\right)-\mathbf{u}_{\tau}(\nu(n))
$$

Observe that $\nu(n)$ converges to $\nu$. Thus, any subsequence also converges to $\nu$. Take, in particular, $\left\{n_{k}\right\}_{k=1}^{\infty}$ such that $n_{k}=k q$. It follows that $\nu\left(n_{k}\right)=\nu$ for all $k$. Finally, after a monotone increasing transformation of utility, we let $n_{k}$ go to infinity and obtain the derivative of the utility with respect to the fraction of votes for $L$

$$
\lim _{n_{k} \rightarrow \infty} \frac{\mathbf{u}_{\tau}\left(\nu+\frac{1}{n_{k}}\right)-\mathbf{u}_{\tau}(\nu)}{1 / n_{k}} \equiv \mathbf{u}_{\tau}^{\prime}(\nu),
$$

resulting in the definition of the benefit of voting presented in the paper

$$
\mathbf{u}_{\tau}^{\prime}(\nu) \equiv \frac{\partial \hat{u}\left(t_{L}, t_{R}, \nu ; \tau\right)}{\partial \nu}=w\left(L ; t_{L}, t_{R}, \nu, \tau\right)
$$

\section{B Proofs of Lemmata}

Lemma 4.1. Let $A 1$ and A2 hold. Then:

(i) Given $t_{L}, t_{R} \in T$ and $\nu \in[0,1]$, everybody votes.

Moreover, for $t_{L}<t_{R}$, everybody to the left of $\hat{t}\left(t_{L}, t_{R}, \nu\right)$ votes for $L$ while everybody to the right of $\hat{t}\left(t_{L}, t_{R}, \nu\right)$ votes for $R$. That is, $V_{L}\left(t_{L}, t_{R}, \nu\right)=$ $\left[\underline{t}, \hat{t}\left(t_{L}, t_{R}, \nu\right)\right)$, and $V_{R}\left(t_{L}, t_{R}, \nu\right)=\left(\hat{t}\left(t_{L}, t_{R}, \nu\right), \bar{t},\right]$.

(ii) $\chi\left(t_{L}, t_{R}\right)$ is non-empty and single-valued for all $\left(t_{L}, t_{R}\right) \in T^{2}$.

(iii) $\chi$ is continuous, except possibly at $t_{L}=t_{R}$.

\footnotetext{
${ }^{28}$ The function $\lfloor x\rfloor$ is known as the floor function, the greatest integer function or the integer part function, and gives the largest integer less than or equal to $x$.
} 
Proof.

(i) That everybody votes follows directly from the definition of $w_{\tau}$. Because, for all $\left(t_{L}, t_{R}, \nu\right)$ and for all $\tau, w\left(L ; t_{L}, t_{R}, \nu, \tau\right)=-w\left(R ; t_{L}, t_{R}, \nu, \tau\right)$ and $w\left(A ; t_{L}, t_{R}, \nu, \tau\right)=$ 0 , then either voting for $L$ or voting for $R$ is at least as preferred as abstaining.

Now, let $t_{L}<t_{R}$ and $t^{0}=\hat{t}\left(t_{L}, t_{R}, \nu\right)$. From (2.1), (2.2) and (2.3):

$$
w\left(L ; t_{L}, t_{R}, \nu, \tau\right)=\frac{\partial u}{\partial t}\left(t^{0} ; \tau\right) \cdot g^{\prime}(\nu) \cdot\left(t_{L}-t_{R}\right)
$$

Thus, $w\left(L ; t_{L}, t_{R}, \nu, \tau\right)>0$ if and only if $\frac{\partial u}{\partial t}\left(t^{0} ; \tau\right)<0$. By single-peakedness, $\frac{\partial u}{\partial t}\left(t^{0} ; \tau\right)<0$ if and only if $\tau<t^{0}$. Hence, everybody to the left of $t^{0}$ votes for $L$ while everybody to the right of $t^{0}$ votes for $R$.

(ii) From (i), $V_{L}\left(t_{L}, t_{R}, \nu\right)=F\left(t^{0}\right)$ and $V_{L}\left(t_{L}, t_{R}, \nu\right)+V_{R}\left(t_{L}, t_{R}, \nu\right)=1$. Thus, we only need to show that there exists a unique solution to the following equation in $\nu$ :

$$
\begin{array}{rlr}
\nu & =F\left(g(\nu) t_{L}+(1-g(\nu)) t_{R}\right) & \text { if } t_{L} \leq t_{R}, \\
\text { or } \quad \nu & =1-F\left(g(\nu) t_{L}+(1-g(\nu)) t_{R}\right) & \text { if } t_{L}>t_{R} .
\end{array}
$$

Consider 3 cases:

Case 1: $t_{L}<t_{R}$. Define $\Psi_{1}(\nu)=F\left(g(\nu) t_{L}+(1-g(\nu)) t_{R}\right)-\nu$. Note that $g \in C^{1}$ (A1), and $F \in C^{1}$. Then $\Psi_{1} \in C^{1}$, and

$$
\Psi_{1}^{\prime}(\nu)=f\left(\hat{t}\left(t_{L}, t_{R}, \nu\right)\right) \cdot g^{\prime}(\nu) \cdot\left(t_{L}-t_{R}\right)-1<0,
$$

since $f \geq 0, g^{\prime} \geq 0$, and $\left(t_{L}-t_{R}\right)<0$. Thus, $\Psi_{1}$ is a continuous, strictly decreasing function. Because $\Psi_{1}(0)=F\left(t_{R}\right)>0$ and $\Psi_{1}(1)=F\left(t_{L}\right)-1<0$, there exists a unique $\nu$ in $(0,1)$ that solves the equation $\Psi_{1}(\nu)=0$.

Case 2: $t_{L}>t_{R}$. Define $\Psi_{2}(\nu)=1-F\left(g(\nu) t_{L}+(1-g(\nu)) t_{R}\right)-\nu$. By symmetry with Case 1 , there exists a unique solution $\nu$ in $(0,1)$ to the equation $\Psi_{2}(\nu)=0$.

Case 3: $t_{L}=t_{R}=t$. For any $\nu \in[0,1], \hat{t}(t, t, \nu)=t$. Thus $\chi(t, t)=1$ is the unique solution we are looking for.

(iii). Let $t_{L}<t_{R}$. We know from (ii) that $\Psi_{1}^{\prime}(\nu) \neq 0$. By the Implicit Function Theorem there exists an open ball $B_{\epsilon}(\check{t})$ of radius $\epsilon$ and center $\check{t}=\left(\check{t}_{L}, \check{t}_{R}\right)$, and a differentiable function $\psi_{\breve{t}}: B_{\epsilon}(\check{t}) \rightarrow \mathbb{R}$, such that if $\left(t_{L}, t_{R}\right) \in B_{\epsilon}(\check{t})$, then

$$
\Psi_{1}\left(\psi_{\breve{t}}\left(t_{L}, t_{R}\right) ; t_{L}, t_{R}\right)=0
$$

Because, from (ii), the solution to " $\Psi\left(\nu, t_{L}, t_{R}\right)=0$ " is unique, $\chi\left(t_{L}, t_{R}\right)$ is a singleton and, thus, can be viewed as a function. Then, (B.1) implies that $\chi\left(t_{L}, t_{R}\right)=$ $\psi_{\check{t}}\left(t_{L}, t_{R}\right)$ for all $\left(t_{L}, t_{R}\right) \in B_{\epsilon}(\check{t})$, i.e., $\psi_{\check{t}}$ is the restriction of $\chi$ to $B_{\epsilon}(\check{t})$. Thus, $\chi$ is 
continuous on $B_{\epsilon}(\check{t})$ and, in particular, at $\check{t}$. Since this is true for all $\left(\check{t}_{L}, \check{t}_{R}\right)$ with $\check{t}_{L}<\check{t}_{R}$, we conclude that $\chi$ is continuous on $\left\{\left(t_{L}, t_{R}\right) \in T^{2}: t_{L}<t_{R}\right\}$. A similar argument applies for $t_{L}>t_{R}$.

Lemma 4.2. The functions $\tilde{t}$ and $\tilde{\pi}_{J}$ are continuous on $T^{2}$.

Proof. Recall that $\tilde{t}\left(t_{L}, t_{R}\right)=g\left(\chi\left(t_{L}, t_{R}\right)\right) t_{L}+\left(1-g\left(\chi\left(t_{L}, t_{R}\right)\right)\right) t_{R}$. By A1, $g$ is a continuous function and, by Lemma 4.1(iii), $\chi$ is continuous for $t_{L} \neq t_{R}$. Thus, $\tilde{t}$ is continuous for $t_{L} \neq t_{R}$.

Let $\bar{t} \in T$, and $\left(t_{L}^{k}, t_{R}^{k}\right) \rightarrow(\bar{t}, \bar{t})$ as $k \rightarrow \infty$. It is easy to see that $\tilde{t}\left(t_{L}^{k}, t_{R}^{k}\right) \rightarrow \hat{t}(\bar{t}, \bar{t})$. Observe that, $\lim _{k \rightarrow \infty} \chi\left(t_{L}^{k}, t_{R}^{k}\right)=F(\bar{t})$, and $\tilde{t}\left(t_{L}^{k}, t_{R}^{k}\right)=t_{R}^{k}+g\left(\chi\left(t_{L}^{k}, t_{R}^{k}\right)\right)\left(t_{L}^{k}-t_{R}^{k}\right)$. Then, $\lim _{k \rightarrow \infty} \tilde{t}\left(t_{L}^{k}, t_{R}^{k}\right)=\bar{t}=\tilde{t}(\bar{t}, \bar{t})$. It follows that $\tilde{t}$ is continuous for $t_{L}=t_{R}$ as well. Hence, $\tilde{t}$ is continuous on $T^{2}$.

Finally, since $\tilde{t}$ and $\pi_{J}$ are continuous, we have that $\tilde{\pi}_{J}\left(t_{L}, t_{R}\right)=\pi_{J}\left(\tilde{t}\left(t_{L}, t_{R}\right)\right)$ is also a continuous function on $T^{2}$.

Lemma 4.3. Let $A 1$ and A2 hold.

(i) The best-response correspondence of party $J, B R_{J}$, is single-valued and continuous, $J=L, R$.

(ii) Given $t_{R} \geq \tau_{L}$, either $L$ can implement its ideal policy, or its best response is $\underline{t}$. That is, either there exists $t_{L}^{o}$ such that $\tilde{t}\left(t_{L}^{o}, t_{R}\right)=\tau_{L}$, or $B R_{L}\left(t_{R}\right)=\{\underline{t}\}$.

(iii) Given $t_{L} \leq \tau_{R}$, either $R$ can implement its ideal policy, or its best response is $\bar{t}$. That is, either there exists $t_{R}^{o}$ such that $\tilde{t}\left(t_{L}, t_{R}^{o}\right)=\tau_{R}$, or $B R_{R}\left(t_{L}\right)=\{\bar{t}\}$.

Proof. (i) We only prove the lemma for party $L$. The proof for party $\mathrm{R}$ is symmetric.

1. Claim: If $t^{\prime}, t^{\prime \prime} \in B R_{L}\left(t_{R}\right)$, then $\tilde{t}\left(t^{\prime}, t_{R}\right)=\tilde{t}\left(t^{\prime \prime}, t_{R}\right)$. Suppose not, say $\tilde{t}\left(t^{\prime}, t_{R}\right)<\tilde{t}\left(t^{\prime \prime}, t_{R}\right)$. By the strict quasi-concavity of $\pi_{L}: \tilde{t}\left(t^{\prime}, t_{R}\right)<\tau_{L}<$ $\tilde{t}\left(t^{\prime \prime}, t_{R}\right)$. But $\tilde{t}$ is a continuous function (Lemma 4.2). Thus, there exists a $t^{\prime \prime \prime}$ between $t^{\prime}$ and $t^{\prime \prime}$ such that $\tilde{t}\left(t^{\prime \prime \prime}, t_{R}\right)=\tau_{L}$, i.e. $\tilde{\pi}_{L}\left(t^{\prime \prime \prime}, t_{R}\right)=\pi_{L}\left(\tau_{L}\right)>$ $\tilde{\pi}_{L}\left(t^{\prime}, t_{R}\right)$, contradicting the fact that $t^{\prime}$ is a best response.

2. It is easy to see that $B R_{L}\left(\tau_{L}\right)=\left\{\tau_{L}\right\}$, since $\tilde{t}\left(t, \tau_{L}\right) \neq \tau_{L}$ for all $t \neq \tau_{L}$.

3. Let $t_{R}>\tau_{L}$ and assume that $t^{\prime}$ and $t^{\prime \prime}$ are best responses for party $L$ to $t_{R}$, with $t^{\prime} \neq t^{\prime \prime}$. Assume, without loss of generality, that $t^{\prime}<t^{\prime \prime}$. Then $t^{\prime \prime}<t_{R}$. Otherwise party $L$ would be better-off by choosing $t_{R}: \tau_{L}<\tilde{t}\left(t_{R}, t_{R}\right)=t_{R}<$ $\tilde{t}\left(t^{\prime \prime}, t_{R}\right)$.

We compute the derivative of $\tilde{t}$ w.r.t. $t_{L}$ as

$$
\frac{\partial \tilde{t}}{\partial t_{L}}\left(t_{L}, t_{R}\right)=-g^{\prime}\left(\chi\left(t_{L}, t_{R}\right)\right) \frac{\partial \chi\left(t_{L}, t_{R}\right)}{\partial t_{L}} \cdot\left(t_{R}-t_{L}\right)+g\left(\chi\left(t_{L}, t_{R}\right)\right),
$$


where $\chi$ is implicitly defined as the unique solution of $F\left(g(\nu) t_{L}+(1-g(\nu)) t_{R}\right)-$ $\nu=0$ (see the proof of Lemma 4.1(ii)). Then, using the fact that

$$
\frac{\partial \chi}{\partial t_{L}}\left(t_{L}, t_{R}\right)=\frac{f(\tilde{t}) g\left(\chi\left(t_{L}, t_{R}\right)\right)}{f(\tilde{t}) g^{\prime}\left(\chi\left(t_{L}, t_{R}\right)\right)\left(t_{R}-t_{L}\right)+1},
$$

we can conclude that

$$
\frac{\partial \tilde{t}}{\partial t_{L}}\left(t_{L}, t_{R}\right)=\frac{g\left(\chi\left(t_{L}, t_{R}\right)\right)}{f\left(\tilde{t}\left(t_{L}, t_{R}\right)\right) g^{\prime}\left(\chi\left(t_{L}, t_{R}\right)\right)\left(t_{R}-t_{L}\right)+1}>0 .
$$

But this implies that $\tilde{t}\left(t^{\prime}, t_{R}\right)<\tilde{t}\left(t^{\prime \prime}, t_{R}\right)$, a contradiction with step 1 .

4. A similar argument applies to $t_{R}<\tau_{L}$. Thus, the best-response correspondence is single-valued.

5. Continuity is a direct implication of the theorem of the maximum.

(ii). If $t_{L}^{o}$ exists, then it is a global maximizer of $\tilde{\pi}_{L}\left(t, t_{R}\right)$ and clearly the best choice for party $L$. If there is no $t \in T$ such that $\tilde{t}\left(t, t_{R}\right)=\tau_{L}$, then it follows from the continuity of $\tilde{t}$ that $\tilde{t}\left(t, t_{R}\right)>\tau_{L}$ for all $t \in T$ (recall that $\left.\tilde{t}\left(t_{R}, t_{R}\right)=t_{R}>\tau_{L}\right)$. Because $\tilde{t}$ is monotone increasing in $t_{L}$ (see step 3 above) and $\pi_{L}$ is single-peaked, it follows that $\tau_{L}<\tilde{t}\left(\underline{t}, t_{R}\right)<\tilde{t}\left(t, t_{R}\right)$ for all $t>\underline{t}$. Thus, $B R_{L}\left(t_{R}\right)=\underline{t}$.

(iii). The proof is a symmetric replica of (ii).

Lemma 5.1. Let $t_{L} \neq t_{R}$. Then $V_{L}$ and $V_{R}$ are continuous functions, $V_{L}$ is nonincreasing in $\nu$, and $V_{R}$ is non-decreasing in $\nu$.

Proof. ¿ ¿From Theorem 5.1, party $L$ 's voters concentrate in the interval $L\left(t_{L}, t_{R}, \nu\right)$. Hence we can write

$$
V_{L}\left(t_{L}, t_{R}, \nu\right)=F\left(\lambda_{2}\left(t_{L}, t_{R}, \nu\right)\right)-F\left(\lambda_{1}\left(t_{L}, t_{R}, \nu\right)\right),
$$

where $\lambda_{2}$ is implicitly defined as the unique $\tau>t_{L}$ that solves $v\left(L ; t_{L}, t_{R}, \nu, \tau\right)=0$. Then, by the Implicit Function Theorem, $\lambda_{2}$ is continuous and

$$
\frac{\partial \lambda_{2}}{\partial \nu}\left(t_{L}, t_{R}, \nu\right)=-\frac{\frac{\partial v}{\partial \nu}\left(L ; t_{L}, t_{R}, \nu, \tau\right)}{\frac{\partial v}{\partial \tau}\left(L ; t_{L}, t_{R}, \nu, \tau\right)}
$$

evaluated at $\tau=\lambda_{2}$. Because, at $\tau=\lambda_{2}$,

$$
\begin{aligned}
& \frac{\partial v}{\partial \nu}\left(L ; t_{L}, t_{R}, \nu, \tau\right)=\frac{\partial w}{\partial \nu}\left(L ; t_{L}, t_{R}, \nu, \tau\right)=u^{\prime \prime}(\hat{t})\left(\frac{\partial \hat{t}\left(t_{L}, t_{R}, \nu\right)}{\partial \nu}\right)^{2}<0, \text { and } \\
& \frac{\partial v}{\partial \tau}\left(L ; t_{L}, t_{R}, \nu, \tau\right)=\frac{\partial w}{\partial \tau}\left(L ; t_{L}, t_{R}, \nu, \tau\right)-\frac{\partial \eta}{\partial \tau}\left(t_{L} ; \tau\right)<0
\end{aligned}
$$




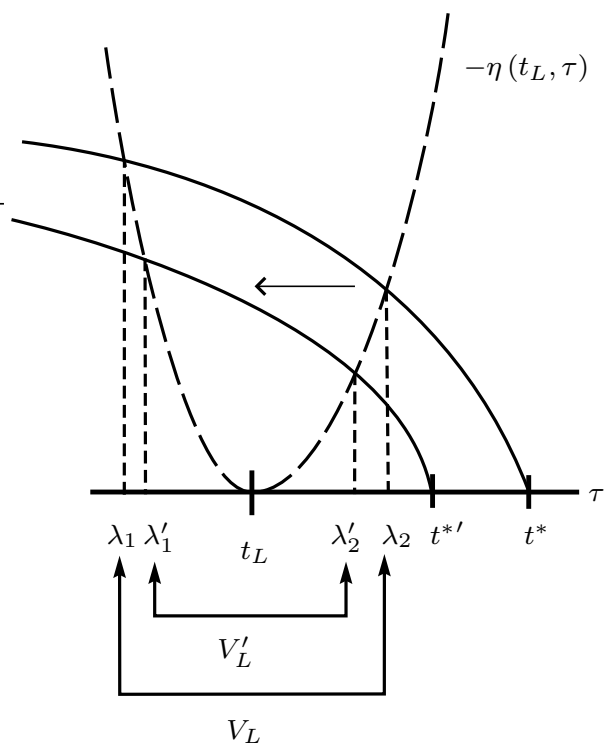

Figure 7: As $\nu$ increases, the support for $L$ decreases.

then $\frac{\partial \lambda_{2}}{\partial \nu}\left(t_{L}, t_{R}, \nu\right)<0$ (see Fig 7.) That is, $\lambda_{2}$ decreases as $\nu$ increases.

If $\lambda_{1}=0$, it cannot decrease any further. If $\lambda_{1}>0$, then it is continuous and, for $\tau=\lambda_{1}$,

$$
\frac{\partial \lambda_{1}}{\partial \nu}\left(t_{L}, t_{R}, \nu\right)=-\frac{\frac{\partial w}{\partial \nu}\left(L ; t_{L}, t_{R}, \nu, \tau\right)}{\frac{\partial w}{\partial \tau}\left(L ; t_{L}, t_{R}, \nu, \tau\right)-\frac{\partial \eta}{\partial \tau}\left(t_{L} ; \tau\right)}>0 .
$$

First, the numerator is negative: $\frac{\partial w}{\partial \nu}<0$. Second, the denominator is positive: $\frac{\partial w}{\partial \tau}<0, \frac{\partial \eta}{\partial \tau}=\zeta^{\prime}>0$ and $\left|\frac{\partial w}{\partial \tau}\right|<\left|\frac{\partial \eta}{\partial \tau}\right|$, since $w$ is weakly concave in $\tau, \eta$ is convex in $\tau$ and $w\left(t_{L}, \lambda_{1}\right)=\eta\left(t_{L}, \lambda_{1}\right)$ (see Fig 7 ). Thus $\frac{\partial \lambda_{1}}{\partial \nu}>0$.

It follows that

$$
\frac{\partial V_{L}}{\partial \nu}=f\left(\lambda_{2}\right) \frac{\partial \lambda_{2}}{\partial \nu}-f\left(\lambda_{1}\right) \frac{\partial \lambda_{1}}{\partial \nu} \leq 0
$$

Similar calculations for $V_{R}$ obtain that $V_{R}$ is non-decreasing in $\nu$.

Lemma 5.2. The consistent voting outcome correspondence $\chi$ is non-empty, singlevalued, and continuous for all $t_{L} \neq t_{R}$.

Proof. Take $t_{L} \neq t_{R}$. From Lemma 5.1 and Theorem 5.1, $V_{L}$ and $V_{R}$ are continuous and $V_{L}\left(t_{L}, t_{R}, \nu\right)+V_{R}\left(t_{L}, t_{R}, \nu\right)>0$. Define

$$
\Psi\left(\nu ; t_{L}, t_{R}\right)=\frac{V_{L}\left(t_{L}, t_{R}, \nu\right)}{V_{L}\left(t_{L}, t_{R}, \nu\right)+V_{R}\left(t_{L}, t_{R}, \nu\right)}-\nu
$$

We know that $\nu \in \chi\left(t_{L}, t_{R}\right)$ if and only if $\Psi\left(\nu ; t_{L}, t_{R}\right)=0$ (see (2.6)), that $\Psi$ is continuous and decreasing (Lemma 5.1$)$, that $\Psi\left(0 ; t_{L}, t_{R}\right) \geq 0$, and that 
$\Psi\left(1 ; t_{L}, t_{R}\right) \leq 0$. Thus, there exists a unique $\nu$ such that $\Psi\left(\nu ; t_{L}, t_{R}\right)=0$. That is, $\chi$ is non-empty and single-valued. Continuity follows directly from the Implicit Function Theorem.

\section{References}

Alesina, A. and Rosenthal, H. (1995). Partisan Politics, Divided Government, and the Economy. Cambridge University Press, Cambridge.

Alesina, A. and Rosenthal, H. (1996). A theory of divided government. Econometrica, 64(6):1311-1341.

Alesina, A. and Rosenthal, H. (2000). Polarized platforms and moderate policies with checks and balances. Journal of Public Economics, 75:1-20.

Ansolabehere, S. and Snyder, Jr., J. M. (2000). Valence politics and equilibrium in spatial election models. Public Choice, 103:327-336.

Aragonés, E. and Palfrey, T. (2002). Mixed equilibrium in a downsian model with a favored candidate. Journal of Economic Theory, 103(1):131-161.

Austen-Smith, D. (1989). Sincere voting in models of legislative elections. Social Choice and Welfare, 6(4):287-299.

Austen-Smith, D. and Banks, J. (1998). Elections, coalitions and legislative outcomes. American Political Science Review, 82:405-422.

Bernhardt, M. D. and Ingberman, D. E. (1985). Candidate reputations and the 'incumbency effect'. Journal of Public Economics, 27:46-67.

Budge, I. and Hofferbert, R. I. (1990). Mandates and policy outputs: U.S. party platforms and federal expenditure. American Political Science Review, 84(1):111131.

Budge, I. and Hofferbert, R. I. (1992). The party mandate and the Westminster model: Election programmes and government spending in Britain. British Journal of Political Science, 22:151-182.

Calvert, R. L. (1985). Robustness of the multidimensional voting model: Candidate motivations, uncertainty, and convergence. American Journal of Political Science, 29:69-95. 
De Sinopoli, F. and Iannantuoni, G. (2003). A spatial voting model where proportional rule leads to two-party equilibria. CEIS Tor Vergata - Research Paper Series No. 31.

Dixit, A., Grossman, G. M., and Gul, F. (2000). A theory of political compromise. Journal of Political Economy, 108(3):531-568.

Ellman, M. and Wantchekon, L. (2000). Electoral competition under the threat of political unrest. Quarterly Journal of Economics, 115(2):499-531.

Fiorina, M. (1974). Representatives, Roll Calls, and Constituences. D. C. Heath, Lexington, MA.

Fudenberg, D. and Tirole, J. (1991). Game Theory. MIT Press, Cambridge, Massachusetts.

Gerber, A. and Ortuño-Ortín, I. (1998). Political compromise and endogenous formation of coalitions. Social Choice and Welfare, 13(3):445-454.

Green, D., Schickler, E., and Palmquist, B. (2002). Partisan Hearts and Minds: Political Parties and the Social Identities of Voters. Yale University Press, New Haven.

Grossman, G. M. and Helpman, E. (1996). Electoral competition and special interest politics. Review of Economic Studies, 63(2):265-286.

Hofferbert, R. I. and Klingemann, H.-D. (1990). The policy impact of party programs and government declarations in the Federal Republic of Germany. European Journal of Political Research, 18:277-304.

King, G., Laver, M., Hofferbert, R. I., Budge, I., and McDonald, M. D. (1993). Party platforms, mandates, and government spending. American Political Science Review, 87(3):744-750.

Klingermann, H.-D., Hofferbert, R. I., Budge, I., Keman, H., Pétry, F., Bergman, T., and Strom, K. (1994). Parties, Policies and Democracy. Westview Press, Boulder, Colo.

Kollman, K., Miller, J. H., and Page, S. E. (1992). Adaptive parties in spatial elections. American Political Science Review, 86(4):929-937.

Lizzeri, A. and Persico, N. (2001). The provision of public goods under alternative electoral incentives. American Economic Review, 91(1):225-239. 
Llavador, H. G. (2000). Abstention and political competition. Review of Economic Design, 5(4):411-432.

Londregan, J. and Romer, T. (1993). Polarization, incumbency, and the personal vote. In Barnett, W. A., Hinich, M. J., and Schofield, N. J., editors, Political Economy: Institutions, Competition, and Representation, chapter 14, pages 355377. Cambridge University Press, Cambridge.

McCarty, N., Poole, K. T., and Rosenthal, H. (2003). Political polarization and income inequality. Mimeo.

Ortuño-Ortín, I. (1997). A spatial model of political competition and proportional representation. Social Choice and Welfare, 14:427-438.

Persson, T. and Tabellini, G. (2000). Political Economics. Explaining Economic Policy. The MIT Press.

Poole, K. T. and Daniels, S. (1985). Ideology, party, and voting in the U.S. congress, 1959-80. American Political Science Review, 79:373-399.

Poole, K. T. and Rosenthal, H. (1984a). The polarization of American politics. Journal of Politics, 46:1061-1079.

Poole, K. T. and Rosenthal, H. (1984b). U.S. presidential elections 1968-1980: A spatial analysis. American Journal of Political Science, 28:283-312.

Poole, K. T. and Rosenthal, H. (1991). Patterns of congressional voting. American Journal of Political Science, 35:228-278.

Poole, K. T. and Rosenthal, H. (1997). Congress: A Political-Economic History of Roll Call Voting. Oxford University Press, New York and Oxford.

Rabinowitz, G. and Macdonald, S. E. (1989). A directional theory of issue voting. American Political Science Review, 83(1):93-121.

Roemer, J. E. (1997). Political-economic equilibrium when parties represent constituents: The unidimensional case. Social Choice and Welfare, 14:479-502.

Roemer, J. E. (2001). Political Competition: Theory and Applications. Harvard University Press, Cambridge, Massachusetts.

Snyder, Jr., J. M. (1994). Safe seats, marginal seats, and party platforms: The logic of platform differentiation. Economics and Politics, 6:201-213. 
Snyder, Jr., J. M. (1996). Constituency preferences: California ballot propositions, 1974-1990. Legislative Studies Quaterly, 21:463-488.

Wittman, D. (1977). Candidates with policy preferences: A dynamic model. Journal of Economic Theory, 14:180-189. 